\title{
Interpreting the galactic center gamma-ray excess in the NMSSM
}

\author{
Junjie Cao, ${ }^{a, b}$ Liangliang Shang, ${ }^{a}$ Peiwen Wu, ${ }^{c}$ Jin Min Yang $^{c}$ and Yang Zhang ${ }^{c}$ \\ ${ }^{a}$ Department of Physics, Henan Normal University, \\ Xinxiang 453007, China \\ ${ }^{b}$ Department of Applied Physics, Xi'an Jiaotong University, \\ Xi'an 710049, China \\ ${ }^{c}$ State Key Laboratory of Theoretical Physics, Institute of Theoretical Physics, Academia Sinica, \\ Beijing 100190, China \\ E-mail: junjiec@itp.ac.cn, shlwell1988@gmail.com, pwwu@itp.ac.cn, \\ jmyang@itp.ac.cn, zhangyang@itp.ac.cn
}

AbSTRACT: In the Next-to-Minimal Supersymmetric Standard Model (NMSSM), all singlet-dominated particles including one neutralino, one CP-odd Higgs boson and one CP-even Higgs boson can be simultaneously lighter than about $100 \mathrm{GeV}$. Consequently, dark matter (DM) in the NMSSM can annihilate into multiple final states to explain the galactic center gamma-ray excess (GCE). In this work we take into account the foreground and background uncertainties for the GCE and investigate these explanations. We carry out a sophisticated scan over the NMSSM parameter space by considering various experimental constraints such as the Higgs data, $B$-physics observables, DM relic density, LUX experiment and the dSphs constraints. Then for each surviving parameter point we perform a fit to the GCE spectrum by using the correlation matrix that incorporates both the statistical and systematic uncertainties of the measured excess. After examining the properties of the obtained GCE solutions, we conclude that the GCE can be well explained by the pure annihilations $\tilde{\chi}_{1}^{0} \tilde{\chi}_{1}^{0} \rightarrow b \bar{b}$ and $\tilde{\chi}_{1}^{0} \tilde{\chi}_{1}^{0} \rightarrow A_{1} H_{i}$ with $A_{1}$ being the lighter singletdominated CP-odd Higgs boson and $H_{i}$ denoting the singlet-dominated CP-even Higgs boson or SM-like Higgs boson, and it can also be explained by the mixed annihilation $\tilde{\chi}_{1}^{0} \tilde{\chi}_{1}^{0} \rightarrow W^{+} W^{-}, A_{1} H_{1}$. Among these annihilation channels, $\tilde{\chi}_{1}^{0} \tilde{\chi}_{1}^{0} \rightarrow A_{1} H_{i}$ can provide the best interpretation with the corresponding $p$-value reaching 0.55 . We also discuss to what extent the future DM direct detection experiments can explore the GCE solutions and conclude that the XENON-1T experiment is very promising in testing nearly all the solutions.

Keywords: Supersymmetry Phenomenology

ARXiv EPRINT: 1506.06471 


\section{Contents}

1 Introduction 1

2 Fitting the GCE in the NMSSM 4

2.1 Theoretical setup for the GCE in the NMSSM 4

$\begin{array}{lll}2.2 & \text { Parameter scan strategy for GCE solution } & 6\end{array}$

3 GCE solutions with $\boldsymbol{H}_{2}$ being the SM-like Higgs boson 7

$\begin{array}{lll}3.1 & \text { Solution I - the } b \bar{b} \text { annihilation channel } & 10\end{array}$

3.2 Solution II - the $H_{1} A_{1}$ annihilation channel 12

$\begin{array}{lll}3.3 & \text { Solution III - the } W^{+} W^{-} \text {annihilation channel } & 13\end{array}$

4 GCE solutions with $H_{1}$ being the SM-like Higgs boson $\quad 14$

$\begin{array}{lll}4.1 & \text { Solution IV - the } H_{1} A_{1} \text { annihilation channel } & 16\end{array}$

$\begin{array}{lll}4.2 & \text { Solution } \mathrm{V} \text { - the } H_{2} A_{1} \text { annihilation channel } & 18\end{array}$

5 Explore the GCE solutions in future DM experiments 18

$\begin{array}{llr}6 & \text { Summary } & 20\end{array}$

A Properties of the singlet-dominated particles $\quad 22$

$\begin{array}{ll}\text { A.1 Neutralino masses and mixings } & 22\end{array}$

A.2 CP-odd Higgs mass matrix 23

A.3 CP-even Higgs mass matrix 24

A.4 Some properties of the singlet-dominated particles 25

\section{Introduction}

The compelling evidences for the existence of Dark Matter (DM) from various cosmological and astrophysical observations have provided us a good portal in the search for new physics beyond the Standard Model (SM). One possible method to explore DM in the present Universe is the indirect detection, which looks for the particles produced when DM annihilates in the DM halo. These particles include photons, antiparticles and neutrinos, and among them gamma rays have often been defined as the golden channel for DM indirect detection since the signal can be traced back to the source. The Large Area Telescope (LAT) onboard the Fermi Gamma-ray Space Telescope, due to its unprecedented angular and energy resolutions, has produced the most detailed maps of the gamma ray sky for a wide range of energies. Intriguingly, as was reported by several independent groups [1-9] and also by Fermi Collaboration itself [10], the Fermi-LAT data have revealed the presence of an extended excess of gamma rays over the modeled foreground and background emissions towards the 
Galactic Center (GC). Although several astrophysical mechanisms, such as the thousands of unresolved millisecond pulsars [11-13] and the interactions between comic rays (CR) and interstellar gases [14-18], have been proposed to interpret this Galactic Center Excess (GCE), they usually fail to generate the morphology and energy spectrum of the GCE simultaneously. ${ }^{1}$ So in this work, we instead consider another possibility that the GCE is produced by the annihilation of DM. Although this interpretation has been constrained by the measurements of $\mathrm{CR}$ such as the Fermi-LAT detection of the gamma-rays from dwarf spheroidal galaxies (dSphs) [21-25], the non-observation of spectral features in the AMS-02 measurements of CR positron [26-29], and PAMELA observations of the CR antiprotons [30-36], it still remains a most attractive one not only because the excess emission shows spectral and morphological properties consistent with a telltale sign from DM annihilation, but also because in such an interpretation, the annihilation cross section required to explain the GCE is of the right size to account for the DM density from thermal freeze-out.

So far there have been a large number of attempts to explain the GCE by DM annihilation in various new physics models [37-108]. In the early analyses of the annihilations, great efforts were focused on the channels $\tilde{\chi} \tilde{\chi} \rightarrow b \bar{b}$ with $m_{\tilde{\chi}} \sim 35 \mathrm{GeV}$ and $\tilde{\chi} \tilde{\chi} \rightarrow \tau \bar{\tau}$ with $m_{\tilde{\chi}} \sim 10 \mathrm{GeV}$ since they can reproduce well the GCE spectrum obtained at that time. Recently a critical reassessment of the DM interpretation was made by examining in a comprehensive way the foreground and background uncertainties [9]. It was found that taking the estimated uncertainty in the high-energy tail of the spectrum into account, a much larger number of DM annihilations are able to fit well the $\gamma$-ray data than previously noted [77, 83]. Explicitly speaking, as far as the annihilation $\tilde{\chi} \tilde{\chi} \rightarrow b \bar{b}$ is concerned, now the mass of $\mathrm{DM}$ is extended to a broader range from $30 \mathrm{GeV}$ to $70 \mathrm{GeV}$ in explaining the GCE $[77,83]$. Other annihilation channels such as DM annihilation into light quark pairs and even gluon pair are also able to provide a good fit to the GCE [83]. More strikingly, this new analysis opens up a very good solution usually neglected before, namely DM annihilation into a pair of light non-standard Higgs bosons [52, 53]. This important progress motivates us to renew the solutions to the GCE in supersymmetric theories, which usually predict the lightest neutralino $\tilde{\chi}_{1}^{0}$ as a natural DM candidate.

As the most economical realization of supersymmetry, the Minimal Supersymmetric Standard Model (MSSM) is unsatisfactory in explaining the GCE due to the following four reasons [70, 98]. First, the relic density of DM has required its mass to be larger than about $40 \mathrm{GeV}[109]$. In this case, the annihilations $\tilde{\chi}_{1}^{0} \tilde{\chi}_{1}^{0} \rightarrow \tau \bar{\tau}, q \bar{q}$ with $q$ denoting a light quark can not provide a good fit any more. Second, except for excessive fine-tuning cases the LHC experiments have pushed the lower mass bounds for the CP-odd Higgs boson and the bottom squarks up to several hundred $\mathrm{GeV}$. As a result, the cross section of DM annihilation into $b \bar{b}$ in present day is too small to significantly contribute to the GCE [70, 98]. Third, due to the small velocity of DM in our galaxy, the annihilation rate for DM into SM-like Higgs pairs is $p$-wave suppressed. Consequently this channel is not large enough to generate the GCE. Finally, as for the annihilations $\tilde{\chi}_{1}^{0} \tilde{\chi}_{1}^{0} \rightarrow W W, Z Z$, their fits to the GCE spectrum indicate that regardless of their annihilation rates the corresponding

\footnotetext{
${ }^{1}$ An exception may be the mechanisms recently proposed in [19, 20].
} 
$p$-values are always less than $0.04[77,83]$. This means that the annihilations can not generate the proper spectrum shape for the GCE. We note that for a given parameter point of the MSSM, DM usually annihilates into multiple final states. In this case, the situation can not be improved greatly because, due to the particle spectrum of the MSSM allowed by the current experiments, either the total cross section falls short for the GCE, or the dominant annihilation channel can not reproduce the GCE spectrum well [97].

Given the problems of the MSSM, we consider to interpret the GCE in the Next-toMinimal Supersymmetric Standard Model (NMSSM) with a $Z_{3}$ symmetry, which is the simplest gauge singlet Higgs extension of the MSSM [110]. Distinguished from the MSSM, the NMSSM predicts three singlet-dominated particles: one neutralino, one CP-even and one CP-odd Higgs bosons. These particles are rather special in that all of them can be simultaneously lighter than about $100 \mathrm{GeV}$, and that the couplings for the interactions among themselves are determined by the parameter $\kappa$, which alone is able to predict the right rates for some annihilation channels to explain the GCE (see the following discussion). These features make the NMSSM with a singlet-dominated DM well suit to account for the GCE because, as we will show below, some golden channels for the GCE need light particles to act as the DM, the mediator and/or the annihilation final state.

We note that the interpretations of the GCE in the NMSSM have been intensively discussed in $[60,61,70,72,73,98]$. However, in $[60,61,70,72,73]$ the authors did not consider the systematic uncertainties mentioned above. As a result, the model parameter space they considered is much narrower than that of this work and the obtained conclusions were incomplete. While for [98], although the authors have taken the uncertainties into account, they considered the parameter space characterized by a large $\lambda$ which is different from our discussion.

The aim of this work is to explore any possible solution to the GCE in the $Z_{3}$ NMSSM. For this end, we perform a sophisticated scan over the model parameters by considering various experimental constraints such as the DM relic density, the Higgs data as well as the observation of dwarf galaxies. We use the correlation matrix presented in [9] to include the systematic uncertainties on the GCE spectrum and only keep the parameter points that can reproduce well the spectrum. In our study we mainly consider a singlino-like DM which is believed to interpret the GCE without excessive fine tuning. As we will show below, the annihilation $\tilde{\chi}_{1}^{0} \tilde{\chi}_{1}^{0} \rightarrow H_{i} A_{1}$ with $H_{i} A_{1}$ denoting a scalar-pseudoscalar Higgs pair may provide the best fit to GCE, and the canonical annihilation $\tilde{\chi}_{1}^{0} \tilde{\chi}_{1}^{0} \rightarrow b \bar{b}$ still remains a satisfactory solution except that $m_{\tilde{\chi}_{1}^{0}}$ is now allowed to vary within a broader range. Moreover, it is interesting to see that the mixed annihilation into $W^{+} W^{-}$and $H_{i} A_{1}$ final states is also able to generate a spectrum consistent with the GCE. These conclusions are quite different from previous studies in the NMSSM.

This paper is organized as follows. In section 2, we introduce some of the characteristic features of NMSSM, the basic knowledge about the GCE and our strategy for the parameter scan. In section 3, we discuss in detail the interpretations of the GCE when $H_{2}$ is the SMlike Higgs boson, and in section 4, we carry out a similar study but for the case that $H_{1}$ acts as the SM-like Higgs boson. We draw our conclusion in section 5 and provide more information of the NMSSM couplings in the appendix. 


\section{Fitting the GCE in the NMSSM}

\subsection{Theoretical setup for the GCE in the NMSSM}

We start our analysis by recapitulating the basics of the NMSSM. As one of the most economical extensions of the MSSM, the NMSSM introduces one gauge singlet Higgs superfield in its matter content, and since one purpose of the extension is to solve the $\mu$-problem of the MSSM, a $Z_{3}$ symmetry is usually adopted in the construction of the superpotential to avoid the appearance of parameters with mass dimension. As a result, the superpotential of the NMSSM and the soft breaking terms in Higgs sector are given by [110]

$$
\begin{aligned}
W^{\mathrm{NMSSM}} & =W_{F}+\lambda \hat{H}_{u} \cdot \hat{H}_{d} \hat{S}+\frac{1}{3} \kappa \hat{S}^{3}, \\
V_{\mathrm{soft}}^{\mathrm{NMSSM}} & =\tilde{m}_{u}^{2}\left|H_{u}\right|^{2}+\tilde{m}_{d}^{2}\left|H_{d}\right|^{2}+\tilde{m}_{S}^{2}|S|^{2}+\left(\lambda A_{\lambda} S H_{u} \cdot H_{d}+\frac{1}{3} \kappa A_{\kappa} S^{3}+\text { h.c. }\right),
\end{aligned}
$$

where $W_{F}$ is the superpotential of the MSSM without the $\mu$-term, $\hat{H}_{u}, \hat{H}_{d}$ and $\hat{S}$ are Higgs superfields with $H_{u}, H_{d}$ and $S$ acting as their scalar components respectively, the dimensionless coefficients $\lambda$ and $\kappa$ parameterize the strengthes of the Higgs self couplings, and $\tilde{m}_{u}, \tilde{m}_{d}, \tilde{m}_{S}, A_{\lambda}$ and $A_{\kappa}$ are soft-breaking parameters. In practice, after the electroweak symmetry breaking the soft-breaking squared masses $\tilde{m}_{u}^{2}, \tilde{m}_{d}^{2}$ and $\tilde{m}_{s}^{2}$ are traded for $m_{Z}$, $\tan \beta \equiv v_{u} / v_{d}$ and $\mu \equiv \lambda v_{s}$ as theoretical inputs.

Due to the presence of the superfield $\hat{S}$, the NMSSM contains a singlino field which is the fermion component of $\hat{S}$, and one more complex Higgs field $S$ compared to the MSSM. As a result, the neutralino mass eigenstates $\tilde{\chi}_{i}^{0}$ (with $i$ ranging from 1 to 5 ) are the mixtures of bino, wino, higgsinos and singlino, and the CP-even (odd) Higgs mass eigenstates $H_{i}$ with $i=1,2,3\left(A_{i}\right.$ with $\left.i=1,2\right)$ are mixtures of the real (imaginary) parts of $H_{u}, H_{d}$ and $S$. Throughout this paper, we assume the mass order $m_{\tilde{\chi}_{1}^{0}}<m_{\tilde{\chi}_{2}^{0}}<\cdots<m_{\tilde{\chi}_{5}^{0}}$ for neutralinos, and $m_{H_{1}}<m_{H_{2}}<m_{H_{3}}, m_{A_{1}}<m_{A_{2}}$ for Higgs bosons.

There are three distinguished features in the NMSSM. One is that DM in the NMSSM may be either singlino-dominated or bino-dominated. As expected, the properties of a singlino-dominated DM are quite different from those of a bino-dominated DM, which makes the DM physics in the NMSSM much richer than that in the MSSM [111]. Another feature is that, in the presence of a singlino-dominated DM with mass below $100 \mathrm{GeV}$, the singlet-dominated CP-even and CP-odd Higgs bosons can be simultaneously lighter than about $100 \mathrm{GeV}[111,112]$, and the strengthes for the interactions among these particles are determined by the parameter $\kappa$ which may be as large as 0.1 . This feature, as we will show below, makes the NMSSM with a singlino-dominated DM well suit to explain the GCE. In the appendix, we list the properties of these particles used in our analysis. The other feature is that either $H_{1}$ or $H_{2}$ in the NMSSM can act as the SM-like Higgs boson [113] and generally speaking, $H_{2}$ as the SM-like boson is more attractive from phenomenological point of view and also from naturalness argument.

In the DM explanation of the GCE, the observed $\gamma$-ray originates mainly from the cascade decays of the annihilation final states. In the NMSSM, the possible annihilation final states include $f \bar{f}, V V, H_{i} H_{j}, A_{i} A_{j}$ and $H_{i} A_{j}$ [114], where $f(V)$ denotes any of the 

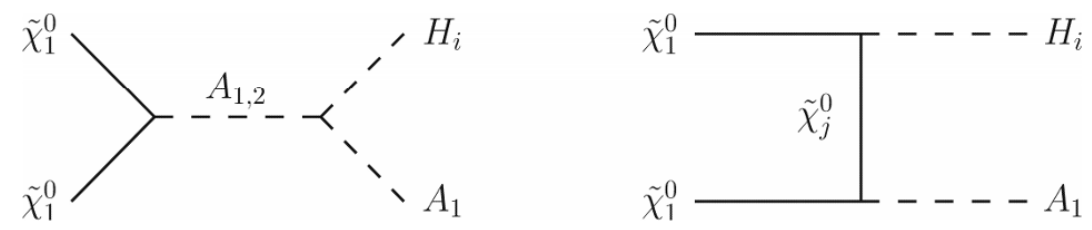

Figure 1. Feynman diagrams contributing to the annihilation $\tilde{\chi}_{1}^{0} \tilde{\chi}_{1}^{0} \rightarrow H_{i} A_{1}$ with $\tilde{\chi}_{j}^{0}(j$ from 1 to 5) denoting any of the five neutralinos. A $u$-channel diagram in associated with the t-channel one is assumed.

fermions (vector bosons) in the SM, and $H_{i}\left(A_{j}\right)$ denotes a CP-even (CP-odd) Higgs boson. In this work, we are particularly interested in the annihilations $\tilde{\chi}_{1}^{0} \tilde{\chi}_{1}^{0} \rightarrow b \bar{b}, W^{+} W^{-}, H_{i} A_{1}$. These annihilations proceed through the $s$-channel mediator of a $\mathrm{Z}$ boson or a Higgs boson with an appropriate $\mathrm{CP}$ quantum number, and also proceed through the $t / u$-channel exchange of a sbottom, a chargino and a neutralino respectively. The complete expressions of the annihilation cross sections are rather complicated, but in non-realistic limit, i.e. the velocity of DM approaching zero, some contributions become unimportant. In this case, the velocity weighted annihilation cross section can be approximated by [114]

$$
\begin{aligned}
\left\langle\sigma_{b \bar{b}} v\right\rangle_{0} \approx & \frac{3 \pi}{2} \sum_{i=1}^{2} \frac{C_{A_{i} \tilde{\chi}_{0}^{0} \tilde{\chi}_{1}^{0}}^{2} C_{A_{i} b \bar{b}}^{2} m_{\tilde{\chi}_{1}^{0}}^{2}}{\left(4 m_{\tilde{\chi}_{1}^{0}}^{2}-m_{A_{i}}^{2}\right)^{2}+m_{A_{i}}^{2} \Gamma_{A_{i}}^{2}}, \\
\left\langle\sigma_{W W} v\right\rangle_{0} \approx & \frac{(\omega-1)^{3 / 2}}{32 \pi m_{\tilde{\chi}_{1}^{0}} m_{W}} \sum_{i=1}^{2}\left(\frac{f_{i, L}^{2}+f_{i, R}^{2}}{1-\omega-k_{i}}\right)^{2}, \\
\left\langle\sigma_{H_{i} A_{1}} v\right\rangle_{0} \approx & \frac{1}{8 \pi}\left(\frac{m_{H_{i}}}{m_{\tilde{\chi}_{1}^{0}}}\right)^{1 / 2}\left(1-\frac{m_{H_{i}}}{2 m_{\tilde{\chi}_{1}^{0}}}\right)^{1 / 2} \sqrt{\delta} \\
& \times\left[\frac{C_{A_{1} A_{1} H_{i}} C_{A_{1} \tilde{\chi}_{1}^{0} \tilde{\chi}_{1}^{0}}}{m_{H_{i}}\left(4 m_{\tilde{\chi}_{1}^{0}}-m_{H_{i}}\right)}+\frac{C_{A_{2} A_{1} H_{i}} C_{A_{2} \tilde{\chi}_{1}^{0} \tilde{\chi}_{1}^{0}}}{4 m_{\tilde{\chi}_{1}^{0}}^{2}-m_{A_{2}}^{2}}+2 \sum_{j=1}^{5} \frac{C_{A_{1} \tilde{\chi}_{1}^{0} \tilde{\chi}_{j}^{0}} C_{H_{i} \tilde{\chi}_{1}^{0} \tilde{\chi}_{j}^{0}}}{m_{H_{i}}+\left|m_{\tilde{\chi}_{j}^{0}}\right|-m_{\tilde{\chi}_{1}^{0}}}\right]^{2}
\end{aligned}
$$

where $C_{X Y Z}$ denotes the coupling of the interaction involving the particles $X, Y$ and $Z$, $\Gamma_{A_{i}}$ is the width of the CP-odd state $A_{i}, \omega=m_{\tilde{\chi}_{1}^{0}}^{2} / m_{W}^{2}, k_{i}=m_{\tilde{\chi}_{i}^{\ddagger}}^{2} / m_{W}^{2}, f_{i, L}\left(f_{i, R}\right)$ is the coupling coefficient for $\tilde{\chi}_{1}^{0} \tilde{\chi}_{i, L}^{ \pm} W^{\mp}\left(\tilde{\chi}_{1}^{0} \tilde{\chi}_{i, R}^{ \pm} W^{\mp}\right)$ interaction, and $\delta \equiv\left(2 m_{\tilde{\chi}_{1}^{0}}-\left(m_{H_{i}}+\right.\right.$ $\left.\left.m_{A_{1}}\right)\right) / 2 m_{\tilde{\chi}_{1}^{0}}$. In getting eq. (2.5), we note that a good fit to the GCE requires that the $H_{i} A_{1}$ final state is produced close to threshold, i.e. $\delta \simeq 0$, so we can expand $\left\langle\sigma_{H_{i} A_{1}} v\right\rangle_{0}$ in terms of $\delta$. Then the first two terms on the right hand of eq. (2.5) come from the left diagram of figure 1, and the last term comes from the right diagram of figure 1.

The flux per unit solid angle at some photon energy $E_{\gamma}$, which is observed by FermiLAT, is then given by

$$
\frac{\mathrm{d} \Phi_{\gamma}\left(E_{\gamma}\right)}{\mathrm{d} E_{\gamma} \mathrm{d} \Omega}=\sum_{X Y} \frac{\left\langle\sigma_{X Y} v\right\rangle_{0}}{8 \pi m_{\tilde{\chi}_{1}^{0}}^{2}} \frac{\mathrm{d} N_{X Y}^{\gamma}}{\mathrm{d} E} \int \mathrm{ds} \rho_{\mathrm{DM}}^{2}(r(s, \theta)),
$$

where $d N_{X Y}^{\gamma} / d E$ is the photon spectrum generated by the annihilation $\tilde{\chi}_{1}^{0} \tilde{\chi}_{1}^{0} \rightarrow X Y, \rho_{D M}$ is the DM profile and the integral over $\rho_{D M}^{2}$ is along the light-of-sight (LOS) at an angle $\theta$ 
towards GC. In the DM interpretation of the GCE, a generalized Navarro, Frenk \& White (NFW) DM profile is usually adopted, and its expression is given by $[115,116]$

$$
\rho(r)=\rho_{\odot}\left(\frac{r}{r_{\odot}}\right)^{-\gamma}\left(\frac{1+r_{\odot} / R_{s}}{1+r / R_{s}}\right)^{3-\gamma}
$$

with slope parameter $\gamma=1.26$, scale radius $R_{s}=20 \mathrm{kpc}$ and the local DM density $\rho_{\odot}=0.4 \mathrm{GeV} / \mathrm{cm}^{3}$ at the radial distance of the sun from the galactic centre $r_{\odot}$. Here the coordinate $r$ is centered on the galactic centre and can be expressed as $r^{2}(s, \theta)=$ $r_{\odot}^{2}+s^{2}-2 r_{\odot} s \cos \theta$ with $s$ and $\theta$ being the LOS distance and the aperture angle between the axis connecting the earth with the galactic centre and the LOS respectively.

In our study, we use the package micrOMEGAs-3.6.9.2 [117-121] to calculate the DM relic density and with the help of PYTHIA [122] to generate the flux in eq. (2.6). Note that in any explicit model, DM usually annihilates into multiple final states. In this case, the different fluxes are summed over.

\subsection{Parameter scan strategy for GCE solution}

We simplify our scan over the NMSSM parameter space by fixing the parameters that are not closely related to the DM studies. The soft SUSY breaking parameters in the squark sector are all fixed to be $2 \mathrm{TeV}$ except that we vary those for the third generation to generate a CP-even Higgs near $125 \mathrm{GeV}$. We assume $A_{t}=A_{b}$ and $M_{U_{3}}=M_{D_{3}}$ to reduce the number of free parameters. Similarly, all of the soft SUSY breaking parameters in the slepton sector are fixed to be $300 \mathrm{GeV}$ to explain the discrepancy of the measured value for muon anomalous magnetic moment from its SM prediction. As for the gaugino sector we abandon the Grand Unified Theory relation and fix the wino mass and gluino mass at $1 \mathrm{TeV}$ and $2 \mathrm{TeV}$ respectively. Consequently, the remained free parameters include $\tan \beta, \mu, \lambda, \kappa, A_{\lambda}, A_{\kappa}$ in the Higgs sector, $M_{Q_{3}}, M_{U_{3}}$ and $A_{t}$ for third generation quarks and the bino mass $M_{1}$, which are all defined at the scale of $2 \mathrm{TeV}$ in the scan. We use NMSSMTools-4.3.0 [123-125] to scan intensively the following NMSSM parameter region:

$$
\begin{aligned}
1 & <\tan \beta<40, & 0 & <\lambda<0.7, \quad 0<|\kappa|<0.7, \\
0 & <A_{\lambda}<5 \mathrm{TeV}, & \left|A_{\kappa}\right| & <2 \mathrm{TeV}, \quad\left|A_{t}\right|<5 \mathrm{TeV}, \\
100 \mathrm{GeV} & <\mu<600 \mathrm{GeV}, & 200 \mathrm{GeV} & <m_{Q_{3}}, m_{U_{3}}<5 \mathrm{TeV} .
\end{aligned}
$$

The process to retain the parameter points include the following steps:

- We require the DM to be singlino-dominated and satisfy $m_{\tilde{\chi}_{1}^{0}} \leq 150 \mathrm{GeV}$, and impose all the experimental constraints encoded in NMSSMTools-4.3.0 [123-125] which include the relic abundance at $3 \sigma$ level $\left(0.107 \leq \Omega h^{2} \leq 0.131\right)$, LUX exclusion bound at $90 \%$ C.L., various B-physics measurements as well as the discrepancy of muon magnetic moment at $2 \sigma$ level. We also consider various electroweak precision data calculated in [126].

- We consider the constraints on the Higgs sector with the package HiggsBounds-4.1.2 [127-130] which contains the data from LEP, Tevetron and LHC. For 
the SM-like Higgs boson, we further perform a fit to the data with the package HiggsSignal [131] and keep the $2 \sigma$ samples.

- We use micrOMEGAs-3.6.9.2 [117-121] to calculate the DM annihilation cross section at present day, and then impose the constraints from dSphs by the data in [25] for the $b \bar{b}$ annihilation channel and with the method introduced in [98] for the $H_{i} A_{1}$ final states.

- We also use micrOMEGAs-3.6.9.2 [117-121] to generate the $\gamma$-ray spectrum. Considering the astrophysical uncertainties which may come from the errors in our setting on the local DM density $\rho_{\odot}$, the scale radius $R_{s}$ and the inner slope parameter $\gamma$ in eq. (2.7), for each parameter point we allow an uncertainty factor $\mathcal{A}$ in the range of $(0.17,5.3)$ for the annihilation cross section, or equivalently for the height of the gamma-ray spectrum in eq. (2.6) [83]. Then for the $\mathcal{A}$-tuned $\gamma$-ray spectrum, we perform a fit to the residual GCE spectrum obtained in [9] by using the publicly available covariance matrix, which include both the statistical and systematic uncertainties of the measured flux. The corresponding $\chi_{s p}^{2}$ function is calculated by $[9,83]$ :

$$
\chi_{s p}^{2}(\mathcal{A})=\sum_{i j}\left(\frac{d \bar{N}}{d E_{i}}-\frac{d N}{d E_{i}}\right) \Sigma_{i j}^{-1}\left(\frac{d \bar{N}}{d E_{j}}-\frac{d N}{d E_{j}}\right)
$$

where $\Sigma_{i j}$ is the covariance matrix, $d N / d E_{i}$ is the measured flux in the $i$-th energy bin, and $d \bar{N} / d E_{i}$ is the flux predicted by the NMSSM, which depends on the parameter point and also on the factor $\mathcal{A}$.

We define the GCE $\chi^{2}$ as the minimum value of $\chi_{s p}^{2}(\mathcal{A})$ among different choices of $\mathcal{A}, \chi_{\mathrm{GCE}}^{2}=\min \left(\chi_{s p}^{2}(\mathcal{A})\right)$, and keep the parameter points that satisfy $\chi_{\mathrm{GCE}}^{2} \leq 35.2$. These points are assumed to have the capability to explain the GCE at $95 \%$ confidence level for 23 degree of freedom [9].

The parameter ranges of the GCE solutions are listed in table 1, which are classified by the dominant final state in DM annihilations (see the following discussion). For the first three types of the DM annihilations $\mathrm{H}_{2}$ acts as the SM-like Higgs boson, while for the last two types $H_{1}$ corresponds to the SM-like Higgs boson. One distinguished feature that table 1 exhibits is that all the singlet dominated particles in the GCE solutions, including DM, the singlet-dominated CP-even and CP-odd Higgs bosons, are lighter than about $150 \mathrm{GeV}$. This feature, as we will emphasized below, makes the NMSSM well suit for explaining the GCE.

\section{GCE solutions with $\boldsymbol{H}_{2}$ being the SM-like Higgs boson}

In this section, we exhibit the features of the GCE solutions for the case that DM is singlino-dominated and $\mathrm{H}_{2}$ acts as the SM-like Higgs boson. All the solutions considered in this work survive the constraints listed in last section and meanwhile can explain the GCE at $95 \%$ C.L.. 


\begin{tabular}{|l|l|l|l|l|l|}
\hline \multirow{2}{*}{} & \multicolumn{3}{|c|}{$H_{2}$ is SM-like } & \multicolumn{1}{c|}{$H_{1}$ is SM-like } \\
\cline { 2 - 6 } & \multicolumn{1}{|c|}{$b \bar{b}$} & \multicolumn{1}{|c|}{$H_{1} A_{1}$} & \multicolumn{1}{|c|}{$W^{+} W^{-}$} & \multicolumn{1}{|c|}{$H_{1} A_{1}$} & $H_{2} A_{1}$ \\
\hline $\tan \beta$ & $(10,30)$ & $(8,40)$ & $(8,40)$ & $(15,20)$ & $(12,20)$ \\
\hline$M_{1}$ & $(-600,-60)$ & $(-600,-80)$ & $(-600,-130)$ & $(-130,-90)$ & $(-200,-100)$ \\
\hline$\lambda$ & $(0.2,0.7)$ & $(0.2,0.6)$ & $(0.2,0.4)$ & $(0.6,0.7)$ & $(0.4,0.7)$ \\
\hline$\kappa$ & $(0.02,0.12)$ & $(0.07,0.15)$ & $(0.09,0.14)$ & $(0.10,0.14)$ & $(0.11,0.16)$ \\
\hline$\mu$ & $(160,300)$ & $(110,210)$ & $(110,160)$ & $(220,270)$ & $(210,270)$ \\
\hline$A_{\lambda}$ & $(2400,5000)$ & $(830,5000)$ & $(970,5000)$ & $(3900,5000)$ & $(2900,5000)$ \\
\hline$A_{\kappa}$ & $(-210,-70)$ & $(-60,22)$ & $(-70,10)$ & $(-65,-16)$ & $(-66,5)$ \\
\hline$A_{t, b}$ & $(-4300,3900)$ & $(-4600,4700)$ & $(-4700,3900)$ & $(-2200,2000)$ & $(-3400,4000)$ \\
\hline$M_{Q_{3}}$ & $(300,5000)$ & $(350,5000)$ & $(500,5000)$ & $(1200,4600)$ & $(700,4800)$ \\
\hline$M_{U_{3}, D_{3}}$ & $(250,5000)$ & $(270,5000)$ & $(400,5000)$ & $(250,5000)$ & $(1400,5000)$ \\
\hline$m_{H_{1}}$ & $(15,102)$ & $(61,119)$ & $(83,110)$ & $(124,127.4)$ & $(124.5,127.4)$ \\
\hline$m_{H_{2}}$ & $(122.8,127.8)$ & $(122.7,128)$ & $(123,128)$ & $(125.6,142)$ & $(125.7,146)$ \\
\hline$\langle\sigma v\rangle_{0}$ & $(0.17,1.9)$ & $(0.29,1.8)$ & $(0.44,1.6)$ & $(0.34,1.2)$ & $(0.38,1.5)$ \\
\hline$m_{\tilde{\chi}_{1}^{0}}$ & $(31,70)$ & $(62,114)$ & $(84,102)$ & $(71,87)$ & $(80,127)$ \\
\hline$m_{\tilde{\chi}_{2}^{0}}$ & $(67,298)$ & $(83,233)$ & $(114,165)$ & $(86,128)$ & $(100,192)$ \\
\hline$m_{\tilde{\chi}_{1}^{ \pm}}$ & $(166,297)$ & $(117,214)$ & $(117,158)$ & $(225,270)$ & $(218,266)$ \\
\hline$m_{A_{1}}$ & $(58,133)$ & $(9,109)$ & $(10,105)$ & $(9,38)$ & $(16,95)$ \\
\hline$m_{H^{ \pm}}$ & $(3510,4666)$ & $(1477,3447)$ & $(2818,2968)$ & $(3883,4941)$ & $(2928,4740)$ \\
\hline$\chi_{\min }^{2}$ & $(23,35)$ & $(21,35)$ & $(24,35)$ & $(21,35)$ & $(21,35)$ \\
\hline & & & & & \\
\hline
\end{tabular}

Table 1. Favored parameter region of the NMSSM to explain the GCE, which are classified by the dominant final state in DM annihilations. These annihilations are called Solution I, II, III, IV and $\mathrm{V}$ respectively in the following discussion. All input parameters are defined at $2 \mathrm{TeV}$ and quantities with mass (annihilation cross section) dimension are in unit of $\mathrm{GeV}\left(10^{-26} \mathrm{~cm}^{3} / \mathrm{s}\right)$.

In figure 2 we project the solutions on $\langle\sigma v\rangle_{0}-m_{\tilde{\chi}_{1}^{0}}$ plane (upper panel) and $\chi_{\mathrm{GCE}}^{2}-m_{\tilde{\chi}_{1}^{0}}$ plane (lower panel). Solutions marked by red square, blue triangle and black asterisk correspond to the cases that DM annihilates with the largest branching ratio into $b \bar{b}, H_{1} A_{1}$ and $W^{+} W^{-}$final states respectively, which hereafter are collectively called Solution I, Solution II and Solution III correspondingly. Then the upper panel of figure 2 indicates that, for the ranges $30 \mathrm{GeV} \leq m_{\tilde{\chi}_{1}^{0}} \leq 40 \mathrm{GeV}, 50 \mathrm{GeV} \leq m_{\tilde{\chi}_{1}^{0}} \leq 62 \mathrm{GeV}$ and $63 \mathrm{GeV} \leq$ $m_{\tilde{\chi}_{1}^{0}} \leq 70 \mathrm{GeV}$, Solution I is viable, while for $63 \mathrm{GeV} \leq m_{\tilde{\chi}_{1}^{0}} \leq 115 \mathrm{GeV}$ and $83 \mathrm{GeV} \leq$ $m_{\tilde{\chi}_{1}^{0}} \leq 100 \mathrm{GeV}$, Solution II and Solution III can account for the GCE respectively. For any of the solutions, the $\langle\sigma v\rangle_{0}$ is larger than $1.7 \times 10^{-27} \mathrm{~cm}^{3} / \mathrm{s}$, and its lower bound increases monotonically as $\tilde{\chi}_{1}^{0}$ becomes heavier. The reason for the latter behavior is that, for a heavier DM, its number density is smaller. So to obtain the same photon flux for the GCE, a larger cross section is needed.

The lower panel of figure 2 indicates that the best interpretation in Solution I comes from $m_{\tilde{\chi}_{1}^{0}} \simeq 50 \mathrm{GeV}$ with $\chi_{\mathrm{GCE}}^{2} \simeq 23$ and a $p$-value of 0.44 . This conclusion coincides with that of [83], which was obtained in a model independent way and for a pure $b \bar{b}$ 

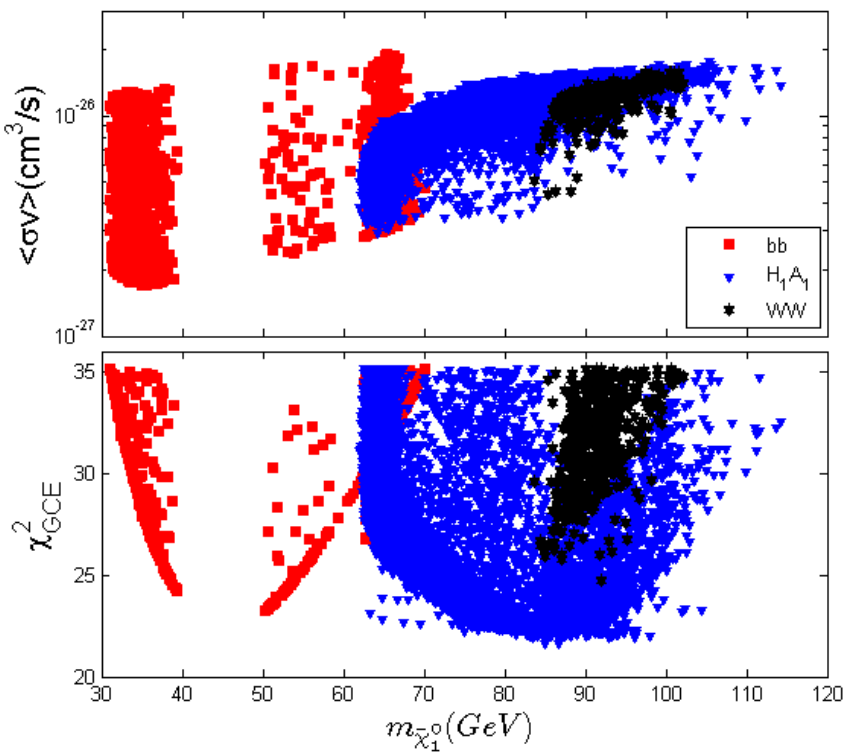

Figure 2. The GCE solutions of the NMSSM for a singlino-dominated DM with $H_{2}$ acting as the SM-like Higgs boson, which are projected on the $\langle\sigma v\rangle_{0}-m_{\tilde{\chi}_{1}^{0}}$ plane (upper panel) and $\chi_{\mathrm{GCE}}^{2}-$ $m_{\tilde{\chi}_{1}^{0}}$ plane (lower panel). Solutions marked by the red square, the blue triangle and the black asterisk correspond to the case that DM annihilates in present day mainly by the channels $\tilde{\chi}_{1}^{0} \tilde{\chi}_{1}^{0} \rightarrow$ $b \bar{b}, H_{1} A_{1}, W^{+} W^{-}$respectively, which are collectively called Solution I, II and III correspondingly.

annihilation channel. For Solutions II and Solutions III, the best interpretations locate at $m_{\tilde{\chi}_{1}^{0}} \simeq 87 \mathrm{GeV}$ with $\chi_{\mathrm{GCE}}^{2} \simeq 21.6$ and $m_{\tilde{\chi}_{1}^{0}} \simeq 92 \mathrm{GeV}$ with $\chi_{\mathrm{GCE}}^{2} \simeq 24.7$ respectively, and the corresponding p-values are 0.54 and 0.36 . These two solutions, within our knowledge, were rarely discussed in previous literatures about the NMSSM. Moreover, we checked that, in the case of $m_{\tilde{\chi}_{1}^{0}} \simeq 40 \sim 50 \mathrm{GeV}\left(m_{\tilde{\chi}_{1}^{0}} \simeq 62 \mathrm{GeV}\right)$, DM annihilated in early universe mainly through a nearly on-shell $Z$ boson (SM-like Higgs boson), and in order to get the correct relic density, the weighted cross section for the annihilation $\tilde{\chi}_{1}^{0} \tilde{\chi}_{1}^{0} \rightarrow A_{i}^{*} \rightarrow b \bar{b}$ was usually less than $1.0 \times 10^{-27} \mathrm{~cm}^{3} \mathrm{~s}^{-1}$. Since nowadays the former annihilation is helicity ( $p$-wave) suppressed, and the rate for the latter annihilation changes little because it proceeds at s-wave level, the $\langle\sigma v\rangle_{0}$ in this range can not reach the size required for the GCE.

In table 2, we present detailed information of three benchmark points P1, P2 and P3 for Solution I, II and III respectively. This table indicates that the sole annihilation channel $\tilde{\chi}_{1}^{0} \tilde{\chi}_{1}^{0} \rightarrow b \bar{b}$ or $\tilde{\chi}_{1}^{0} \tilde{\chi}_{1}^{0} \rightarrow H_{1} A_{1}$ can be responsible for the GCE; while for the channel $\tilde{\chi}_{1}^{0} \tilde{\chi}_{1}^{0} \rightarrow W^{+} W^{-}$, it must mix sizeably with the channel $\tilde{\chi}_{1}^{0} \tilde{\chi}_{1}^{0} \rightarrow H_{1} A_{1}$ to account for the GCE. We will return to this issue later.

In our calculation, we found that the condition on the GCE $\chi^{2}$ can reduce the number of the parameter points that survive the constraints by more than $90 \%$. This implies that the GCE has non-trivial requirements on the parameters of the NMSSM, especially it suggests that some of the independent parameters may be correlated. Motivated by this thought, we study the correlations among the parameters $\lambda, \kappa, \mu, m_{\tilde{\chi}_{1}^{0}}$ and $m_{A_{1}}$ which are important parameters in the interpretation of the GCE and show the corresponding results 


\begin{tabular}{|c|c|c|c|c|c|c|c|c|c|c|}
\hline Point & $\tan \beta$ & $\lambda$ & $\kappa$ & $\mu$ & $A_{\lambda}$ & $A_{\kappa}$ & $A_{D_{3}, U_{3}}$ & $M_{1}$ & $M_{Q_{3}}$ & $M_{U_{3}, D_{3}}$ \\
\hline P1 & 16 & 0.36 & 0.04 & 241 & 3891 & -136 & 420 & -472 & 4127 & 4445 \\
\hline P2 & 12 & 0.46 & 0.12 & 179 & 2036 & -6 & -2354 & -209 & 2197 & 3673 \\
\hline P3 & 13 & 0.27 & 0.11 & 130 & 1899 & -5 & -524 & -170 & 4098 & 4384 \\
\hline P4 & 18 & 0.69 & 0.12 & 243 & 4518 & -43 & -320 & -103 & 1436 & 4308 \\
\hline P5 & 17 & 0.66 & 0.13 & 226 & 3923 & -17 & 1138 & -97 & 4540 & 1286 \\
\hline P6 & 18 & 0.66 & 0.15 & 217 & 4048 & -24 & 2050 & -103 & 4170 & 1452 \\
\hline P7 & 15 & 0.50 & 0.13 & 255 & 4085 & -35 & 2621 & -131 & 2935 & 4468 \\
\hline \hline Pont
\end{tabular}

\begin{tabular}{|c|c|c|c|c|c|c|c|c|c|c|}
\hline Point & $m_{H_{1}}$ & $m_{H_{2}}$ & $m_{H^{ \pm}}$ & $m_{A_{1}}$ & $m_{\tilde{\chi}_{1}^{0}}$ & $m_{\tilde{\chi}_{2}^{0}}$ & $m_{\tilde{\chi}_{1}^{ \pm}}$ & $B r_{\left(h 2 \rightarrow \tilde{\chi}_{1}^{0} \tilde{\chi}_{1}^{0}\right)}$ & $\operatorname{Br}_{\left(h 1 \rightarrow A_{1} A_{1}\right)}$ & $B r_{\left(h 2 \rightarrow A_{1} A_{1}\right)}$ \\
\hline $\mathrm{P} 1$ & 40 & 125 & 3960 & 99 & 50 & 256 & 248 & $0.54 \%$ & 0 & 0 \\
\hline $\mathrm{P} 2$ & 99 & 125 & 2065 & 66 & 87 & 178 & 183 & 0 & 0 & 0 \\
\hline P3 & 99 & 126 & 1823 & 48 & 92 & 126 & 134 & 0 & $88.51 \%$ & $7.06 \%$ \\
\hline $\mathrm{P} 4$ & 126 & 133 & 4452 & 20 & 78 & 102 & 249 & 0 & $6.50 \%$ & $95.32 \%$ \\
\hline P5 & 125 & 126 & 3883 & 27 & 81 & 96 & 231 & 0 & $4.86 \%$ & $94.01 \%$ \\
\hline $\mathrm{P} 6$ & 126 & 129 & 4022 & 33 & 85 & 101 & 222 & 0 & $4.55 \%$ & $95.74 \%$ \\
\hline $\mathrm{P} 7$ & 125 & 145 & 4068 & 69 & 121 & 127 & 262 & 0 & 0 & $94.11 \%$ \\
\hline
\end{tabular}

\begin{tabular}{|c|c|c|c|c|c|c|c|c|c|c|}
\hline \hline Point & $\chi_{\mathrm{GCE}}^{2}$ & p-value & $\langle\sigma v\rangle_{0}$ & $\left.\langle\sigma v\rangle\right|_{T_{F}}$ & $\sigma_{p}^{\mathrm{SI}}$ & $\sigma_{p}^{\mathrm{SD}}$ & $R_{b \bar{b}}$ & $R_{H_{1} A_{1}}$ & $R_{w^{+} w^{-}}$ & $R_{H_{2} A_{1}}$ \\
\hline P1 & 23.3 & 0.44 & $6.1 \mathrm{E}-27$ & $2.8 \mathrm{E}-26$ & $2.3 \mathrm{E}-15$ & $1.5 \mathrm{E}-04$ & $90.4 \%$ & $0.0 \%$ & $0.0 \%$ & $0.0 \%$ \\
\hline P2 & 21.6 & 0.54 & $1.4 \mathrm{E}-26$ & $2.6 \mathrm{E}-26$ & $8.2 \mathrm{E}-10$ & $1.3 \mathrm{E}-03$ & $0.0 \%$ & $96.8 \%$ & $3.1 \%$ & $0.0 \%$ \\
\hline P3 & 24.8 & 0.36 & $1.3 \mathrm{E}-26$ & $2.7 \mathrm{E}-26$ & $1.9 \mathrm{E}-10$ & $8.5 \mathrm{E}-04$ & $0.1 \%$ & $46.1 \%$ & $47.2 \%$ & $0.0 \%$ \\
\hline P4 & 22.4 & 0.50 & $8.6 \mathrm{E}-27$ & $2.8 \mathrm{E}-26$ & $5.5 \mathrm{E}-10$ & $1.6 \mathrm{E}-03$ & $0.1 \%$ & $94.3 \%$ & $0.1 \%$ & $5.4 \%$ \\
\hline P5 & 21.4 & 0.55 & $9.9 \mathrm{E}-27$ & $3.1 \mathrm{E}-26$ & $4.4 \mathrm{E}-10$ & $1.7 \mathrm{E}-03$ & $0.1 \%$ & $68.3 \%$ & $0.2 \%$ & $31.4 \%$ \\
\hline P6 & 21.6 & 0.54 & $8.3 \mathrm{E}-27$ & $3.2 \mathrm{E}-26$ & $9.8 \mathrm{E}-10$ & $2.0 \mathrm{E}-03$ & $0.1 \%$ & $42.1 \%$ & $2.1 \%$ & $55.5 \%$ \\
\hline P7 & 23.7 & 0.42 & $7.9 \mathrm{E}-27$ & $3.3 \mathrm{E}-26$ & $1.1 \mathrm{E}-09$ & $5.5 \mathrm{E}-04$ & $0.1 \%$ & $2.2 \%$ & $7.2 \%$ & $84.0 \%$ \\
\hline
\end{tabular}

Table 2. Detailed information of the benchmark points used in our discussion. Quantities with mass, annihilation and scattering cross section dimension are in unit of $\mathrm{GeV}, \mathrm{cm}^{3} / \mathrm{s}$ and pb respectively.

in figure 3. In the following, we concentrate separately on each kind of the solutions and investigate its features. Such a study is helpful to understand the correlations in figure 3 and also the properties of the benchmark points listed in table 2 .

\subsection{Solution I - the $b \bar{b}$ annihilation channel}

Among the solutions to the GCE, Solution I is the most intensively studied one. After considering the systematic uncertainties, one important improvement of Solution I over its previous version is that DM mass is now allowed in the range from $30 \mathrm{GeV}$ to $70 \mathrm{GeV}$, which is much wider than before.

The key features of Solution I are as follows:

- The lighter CP-odd Higgs boson is correlated with DM by $m_{A_{1}} \simeq 2 m_{\tilde{\chi}_{1}^{0}}$. This correlation is shown in the upper right panel of figure 3 which means that the annihilation proceeds resonantly. 

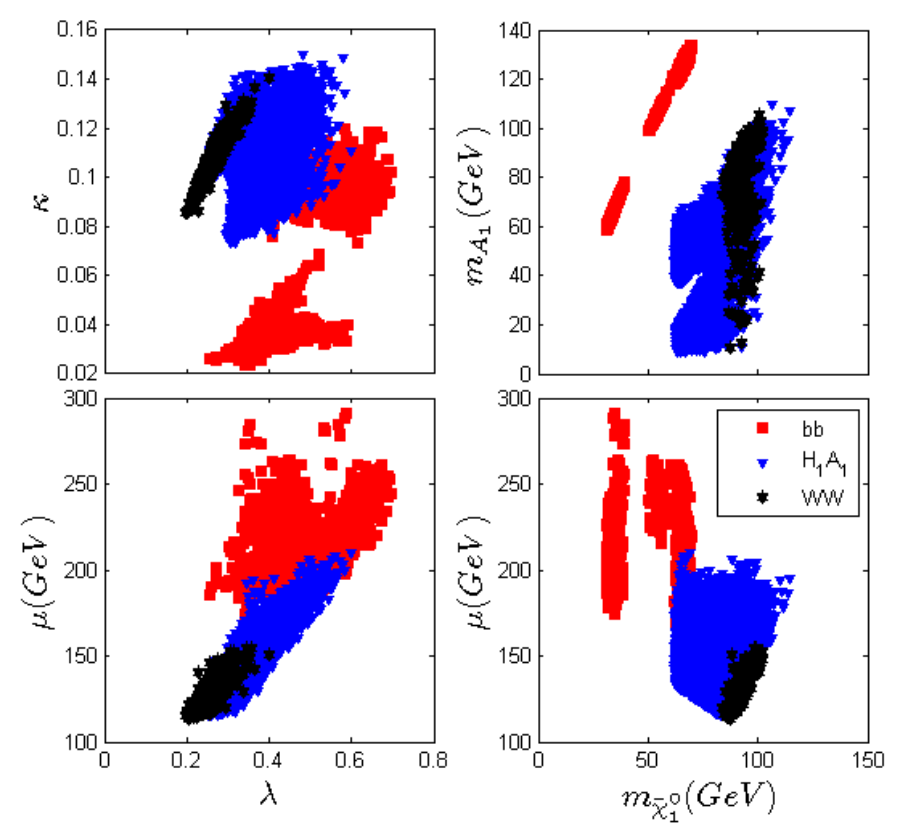

Figure 3. Same as figure 2, but showing the correlations of different parameters.

This feature can be understood as follows. In Solution I, the heavy CP-odd Higgs boson is doublet-dominated with its mass usually at TeV scale. Then eq. (2.3) indicates that the main contribution to the annihilation comes from the moderately light $A_{1}$, which is singlet-dominated. With the formula presented in eq. (A.20) and $v_{s} \equiv \mu / \lambda \gtrsim 450 \mathrm{GeV}$ shown in the lower left panel of figure 3 , one can get

$$
C_{A_{1} \tilde{\chi}_{1}^{0} \tilde{\chi}_{1}^{0}}^{2} C_{A_{1} b \bar{b}}^{2} \simeq \lambda^{2} \kappa^{2}\left(\frac{m_{b}}{\mu}\right)^{2} \lesssim\left(\frac{5}{450}\right)^{2} \kappa^{2}
$$

This inequation means that the couplings involved in the annihilation are highly suppressed so that the process must proceed resonantly to ensure $\left\langle\sigma_{b \bar{b}} v\right\rangle_{0} \sim 10^{-26} \mathrm{~cm}^{3} / \mathrm{s}$. Moreover, our results indicate that the width of $A_{1}$ is very small, $\Gamma_{A_{1}} \lesssim 10^{-2} \mathrm{MeV}$. So as $m_{A_{1}}$ approaches $2 m_{\tilde{\chi}_{1}^{0}}$, the denominator in eq. (2.3) tends to vanish and a small $\kappa$ in eq. (3.1) is then sufficient to predict the right rate of the annihilation for the GCE. This character is illustrated in the upper left panel of figure 3. In fact, a small $\kappa$ is also favored to predict light $\tilde{\chi}_{1}^{0}$ and $A_{1}$, which can be seen from eq. (A.3) and eq. (A.8).

- The parameter $\mu$ is upper bounded by about $300 \mathrm{GeV}$, which is shown in the lower panels of figure 3 .

This feature is actually required by the DM relic density [73]. Generally speaking, in order to predict the measured $\Omega h^{2}$, the velocity weighted cross section $\langle\sigma v\rangle$ should be around the canonical value $3 \times 10^{-26} \mathrm{~cm}^{3} / \mathrm{s}$ at freezing out (see for example points in table 2). Since $2 m_{\chi_{1}^{0}} / m_{A_{1}}>1$ in Solution I, $\langle\sigma v\rangle$ for the annihilation $\tilde{\chi}_{1}^{0} \tilde{\chi}_{1}^{0} \rightarrow A_{1}^{*} \rightarrow b \bar{b}$ at present day is usually larger than that at freezing out due to 
the thermal broadening [132]. Since the dwarf galaxy measurements have required $\left\langle\sigma_{b \bar{b}} v\right\rangle_{0} \lesssim 2 \times 10^{-26} \mathrm{~cm}^{3} / \mathrm{s}$ (see figure 2), new contributions such as those mediated by a $Z$ boson or a CP-even Higgs boson must intervene for the DM annihilation in early Universe, and a moderately small $\mu$ can accelerate the annihilation [73].

- Solution I suffers from severe fine tuning problem. Explicitly speaking, beside the correlation $m_{A_{1}} \simeq 2 m_{\tilde{\chi}_{1}^{0}}$, there exits another strong correlation observed in our analysis, which is given by

$$
m_{\tilde{\chi}_{1}^{0}} / \mathrm{GeV} \simeq \begin{cases}51-475 \kappa, & \text { for } 30 \mathrm{GeV} \leq m_{\tilde{\chi}_{1}^{0}} \leq 40 \mathrm{GeV} \text { or } 0.024 \leq \kappa \leq 0.045, \\ 37+325 \kappa, & \text { for } 50 \mathrm{GeV} \leq m_{\tilde{\chi}_{1}^{0}} \leq 62 \mathrm{GeV} \text { or } 0.038 \leq \kappa \leq 0.07, \\ 49+175 \kappa, & \text { for } 63 \mathrm{GeV} \leq m_{\tilde{\chi}_{1}^{0}} \leq 70 \mathrm{GeV} \text { or } \quad 0.08 \leq \kappa \leq 0.12 .\end{cases}
$$

These correlations make Solution I in the NMSSM quite unnatural to explain the GCE.

- We checked that $\operatorname{Br}\left(A_{1} \rightarrow \gamma \gamma\right)<5 \times 10^{-4}$ so that the $\gamma$-ray spectral line generated by $\tilde{\chi}_{1}^{0} \tilde{\chi}_{1}^{0} \rightarrow A_{1}^{*} \rightarrow \gamma \gamma$ is suppressed.

- Since $\tilde{\chi}_{1}^{0} \lesssim 60 \mathrm{GeV}$ for most cases in Solution I, the SM-like Higgs boson $H_{2}$ may decay into $\tilde{\chi}_{1}^{0}$ pair. We checked that $\operatorname{Br}\left(H_{2} \rightarrow \tilde{\chi}_{1}^{0} \tilde{\chi}_{1}^{0}\right) \lesssim 18 \%$, which is required by the Higgs data at the LHC.

\subsection{Solution II - the $H_{1} A_{1}$ annihilation channel}

Solution II is quite similar to the interpretations presented in $[52,53,88,98,104,106]$, which utilize the process $\tilde{\chi} \tilde{\chi} \rightarrow \phi_{1} \phi_{2} \rightarrow f_{1} \bar{f}_{1} f_{2} \bar{f}_{2}\left(\phi_{1}\right.$ and $\phi_{2}$ denote scalar or pseudoscalar particles, and $f_{1}$ and $f_{2}$ are SM fermions) for the GCE. These interpretations, as were emphasized by the proposers, can easily escape the constraints from DM detection experiments and have been paid more and more attention recently.

The features of Solution II are as follows:

- The singlet-dominated particles satisfy $60 \mathrm{GeV} \lesssim m_{\tilde{\chi}_{1}^{0}} \lesssim 115 \mathrm{GeV}, 10 \mathrm{GeV} \lesssim m_{A_{1}} \lesssim$ $110 \mathrm{GeV}, 60 \mathrm{GeV} \lesssim m_{H_{1}} \lesssim 120 \mathrm{GeV}$ and $\delta<0.2$, and for most samples there exist following relations $m_{H_{1}} \geqslant m_{\tilde{\chi}_{1}^{0}} \geqslant m_{A_{1}}$. Given $\kappa \sim 0.1$ which is required to predict the right size of the annihilation $\tilde{\chi}_{1}^{0} \tilde{\chi}_{1}^{0} \rightarrow H_{1} A_{1}$ for the GCE (see below), the particle spectrum limits parameters such as $\lambda, \mu$ and $A_{\kappa}$ in certain regions (see the expressions of the tree level masses in appendix), which are given in table 1 , and also shown in figure 3.

Note that $\mu$ is below about $200 \mathrm{GeV}$. In this case, the higgsino-dominated neutralinos $\tilde{\chi}_{i}^{0}$ may decay dominantly into $\tilde{\chi}_{1}^{0} A_{1}$ instead of into $\tilde{\chi}_{1}^{0} Z$ since the kinematics is forbidden. In this case, the LHC search for electroweakinos by trilepton $+E_{T}^{\text {miss }}$ signal is less efficient in ruling out the light higgsinos. ${ }^{2}$ Also note that the parameters $\lambda$

\footnotetext{
${ }^{2}$ In doing [111], we once confronted with the situation quite similar to what we are facing now. Our detailed simulation at that time indicated that the trilepton constraint on SUSY is very weak. Moreover, in comparison with the case discussed in [133], we find that our case is more difficult to detect since the signal is smaller.
} 
and $\mu$ are related by $\mu / \mathrm{GeV} \approx 60+260 \lambda$ for $\lambda$ varying from 0.2 to 0.6 (see lower left panel of figure 2), which means that $v_{s} \equiv \mu / \lambda>360 \mathrm{GeV}$. This ensures that the expansions for the masses and couplings in appendix by the power of $\lambda v / \mu$ are good approximations.

- The s-channel contributions to the annihilation rate $\left\langle\sigma_{H_{1} A_{1}} v\right\rangle_{0}$ in eq. (2.5) are usually much smaller than those from the $t / u$ channel, and among the $t / u$ channel contributions, the one induced by the exchange of $\tilde{\chi}_{1}^{0}$ is far dominant. As for the contributions induced by the two higgsino-like neutralinos, each of them may be sizable, but since they cancel each other, the net higgsino contribution is not important. These characters can be understood by the following approximations (see eq. (A.20))

$$
\begin{aligned}
& C_{A_{1} \tilde{\chi}_{1}^{0} \tilde{\chi}_{1}^{0}} C_{H_{1} \tilde{\chi}_{1}^{0} \tilde{\chi}_{1}^{0}} \simeq 2 i \kappa^{2}\left(1+2 \frac{\lambda v}{\mu}\right)^{2}, \\
& C_{A_{1} \tilde{\chi}_{1}^{0} \tilde{\chi}_{i}^{0}} C_{H_{1} \tilde{\chi}_{1}^{0} \tilde{\chi}_{i}^{0}} \simeq\left\{\begin{array}{cl}
-\frac{i}{4} \frac{\lambda^{2} v^{2}}{\mu^{2}} \sin ^{2} \beta, & \text { for Higgsino }- \text { like } \tilde{\chi}_{i}^{0} \text { and } m_{\tilde{\chi}_{i}^{0}}<0, \\
\frac{i}{4} \frac{\lambda^{2} v^{2}}{\mu^{2}} \sin ^{2} \beta, & \text { for Higgsino }- \text { like } \tilde{\chi}_{i}^{0} \text { and } m_{\tilde{\chi}_{i}^{0}}>0,
\end{array}\right.
\end{aligned}
$$

and by the fact that $\kappa \sim 0.1$ is enough to predict the $\tilde{\chi}_{1}^{0}$ contributed $\left\langle\sigma_{H_{i} A_{1}} v\right\rangle_{0}$ at the order of $10^{-26} \mathrm{~cm}^{3} / \mathrm{s}$ (see equation (3.20) in [98]).

- Since $m_{A_{1}} \lesssim 60 \mathrm{GeV}$ for most cases in Solution II (see upper right panel of figure 2), the SM-like Higgs boson $H_{2}$ may decay into $A_{1} A_{1}$ with a sizeable fraction. Given that $A_{1}$ decays dominantly into $b \bar{b}$, this will result in $4 b$ signal for the SM-like Higgs boson. We checked that $\operatorname{Br}\left(H_{2} \rightarrow A_{1} A_{1}\right) \lesssim 24 \%$, where the upper bound comes from the constraints of the LHC Higgs data.

- Since a good fit to the GCE requires that $H_{1} A_{1}$ is produced close to threshold, the annihilation $\tilde{\chi}_{1}^{0} \tilde{\chi}_{1}^{0} \rightarrow H_{1} A_{1}$ will produce spectral line or box-shaped spectrum in $\gamma$-ray [88, 98]. We checked that $\operatorname{Br}\left(H_{1} \rightarrow \gamma \gamma\right) \leq 1 \times 10^{-3}$ for most samples and $\operatorname{Br}\left(A_{1} \rightarrow \gamma \gamma\right)<4 \times 10^{-4}$ for all samples. So current results of the Fermi-LAT search for spectral lines [134] can not impose tight limit on Solution II (see [88] for a detailed discussion).

\subsection{Solution III - the $W^{+} W^{-}$annihilation channel}

In general, the pure annihilation $\tilde{\chi}_{1}^{0} \tilde{\chi}_{1}^{0} \rightarrow W^{+} W^{-}$is unable to explain the GCE quite well $[77,83]$, but if it mixes sizably with other annihilation channels, the generated spectrum may be improved significantly to account for the GCE. Solution III in the NMSSM belongs to this case.

Solution III has the following features:

- The $W$ pair must be produced close to threshold to account for the GCE, which means $85 \mathrm{GeV} \lesssim m_{\tilde{\chi}_{1}^{0}} \lesssim 100 \mathrm{GeV}$ (see right panels of figure 3 ).

- From the expression of $\left\langle\sigma_{W W} v\right\rangle_{0}$ in eq. (2.4), one can learn that, if the wino is decoupled, the annihilation rate is determined by the higgsino-dominated chargino. 
In this case, we have

$$
\begin{aligned}
& f_{1, L} \simeq-\frac{g}{\sqrt{2}} N_{14} \simeq \frac{g}{\sqrt{2}} \frac{\lambda v}{\mu} \sin \beta \simeq \sqrt{2} g \sin \beta \frac{\kappa v}{m_{\tilde{\chi}_{1}^{0}}}, \\
& f_{1, R} \simeq-\frac{g}{\sqrt{2}} N_{13} \simeq-\sqrt{2} g \sin \beta \frac{\kappa v}{\mu} .
\end{aligned}
$$

In getting these expressions, we note $v_{s} \equiv \mu / \lambda \gtrsim 400 \mathrm{GeV}$ (see lower left panel of figure 2), and expand $N_{13}$ and $N_{14}$ in terms of $\lambda v / \mu$ (see appendix). We also use the approximation $m_{\tilde{\chi}_{1}^{0}} \simeq 2 \kappa \mu / \lambda$. Then $\left\langle\sigma_{W W} v\right\rangle_{0} \sim 10^{-26} \mathrm{~cm}^{3} / s$ and $m_{\tilde{\chi}_{1}^{0}} \sim 90 \mathrm{GeV}$ limit tightly the ranges of the parameters $\lambda, \kappa$ and $\mu$, which are shown in table 1 and figure 3 .

Note in Solution III, the parameter $\mu$, or equivalently the masses for the higgsinodominated chargino and neutralinos, is less than about $150 \mathrm{GeV}$. Since the splitting between $\mu$ and $m_{\tilde{\chi}_{1}^{0}}$ is less than about $50 \mathrm{GeV}$, such a low value of $\mu$ is still allowed by the LHC search for SUSY (see footnote 2 in our discussion on Solution II).

- The upper left panel of figure 3 indicates that the parameters $\lambda$ and $\kappa$ are correlated by

$$
\kappa \simeq 0.03+0.3 \lambda, \quad \text { for } 0.2 \leq \lambda \leq 0.4 \text {. }
$$

As a result, we have $m_{\tilde{\chi}_{1}^{0}} \simeq 2 \mu / 3$.

- As we emphasized before, the annihilation $\tilde{\chi}_{1}^{0} \tilde{\chi}_{1}^{0} \rightarrow W^{+} W^{-}$must mix sizably with the annihilation $\tilde{\chi}_{1}^{0} \tilde{\chi}_{1}^{0} \rightarrow H_{1} A_{1}$ to explain the GCE. This, in return, requires appropriate masses for $H_{1}$ and $A_{1}$ to improve the $\gamma$-ray spectrum generated by the $W W$ state. In figure 4, we plot the GCE $\chi^{2}$ as a function of DM mass in Solution III with different colors denoting the branching ratio of the DM annihilation into $H_{1} A_{1}$. This figure indicates that, with the increase of the branching ratio, the GCE $\chi^{2}$ tends to decrease.

\section{GCE solutions with $H_{1}$ being the SM-like Higgs boson}

In this section, we investigate the GCE solutions for the case that DM is singlino-dominated, and meanwhile $H_{1}$ acts as the SM-like Higgs boson. We carry out our study in a way similar to what we did in section 3 .

In figure 5 we project the solutions on $\langle\sigma v\rangle_{0}-m_{\tilde{\chi}_{1}^{0}}$ plane (upper panel) and $\chi_{\mathrm{GCE}}^{2}-m_{\tilde{\chi}_{1}^{0}}$ plane (lower panel). For solutions marked by green lozenge, DM annihilates with the largest branching ratio into $H_{1} A_{1}$, while for those marked by red pentastar, DM annihilates mainly into $H_{2} A_{1}$. In the following, we call these two kinds of solutions Solution IV and Solution $\mathrm{V}$ respectively. Figure 5 then indicates that, for $70 \mathrm{GeV} \leq m_{\tilde{\chi}_{1}^{0}} \leq 87 \mathrm{GeV}$, Solution IV can explain the GCE quite well with the best explanation coming from $m_{\tilde{\chi}_{1}^{0}} \simeq 81 \mathrm{GeV}$ with $\chi_{\mathrm{GCE}}^{2} \simeq 21.4$ (corresponding to a p-value of 0.55 ), and for $80 \mathrm{GeV} \leq m_{\tilde{\chi}_{1}^{0}} \leq 130 \mathrm{GeV}$, Solution $\mathrm{V}$ is good in accounting for the GCE with the best explanation locating at $m_{\tilde{\chi}_{1}^{0}} \simeq$ $85 \mathrm{GeV}$ with $\chi_{\mathrm{GCE}}^{2} \simeq 21.6$ and a p-value of 0.54 . 


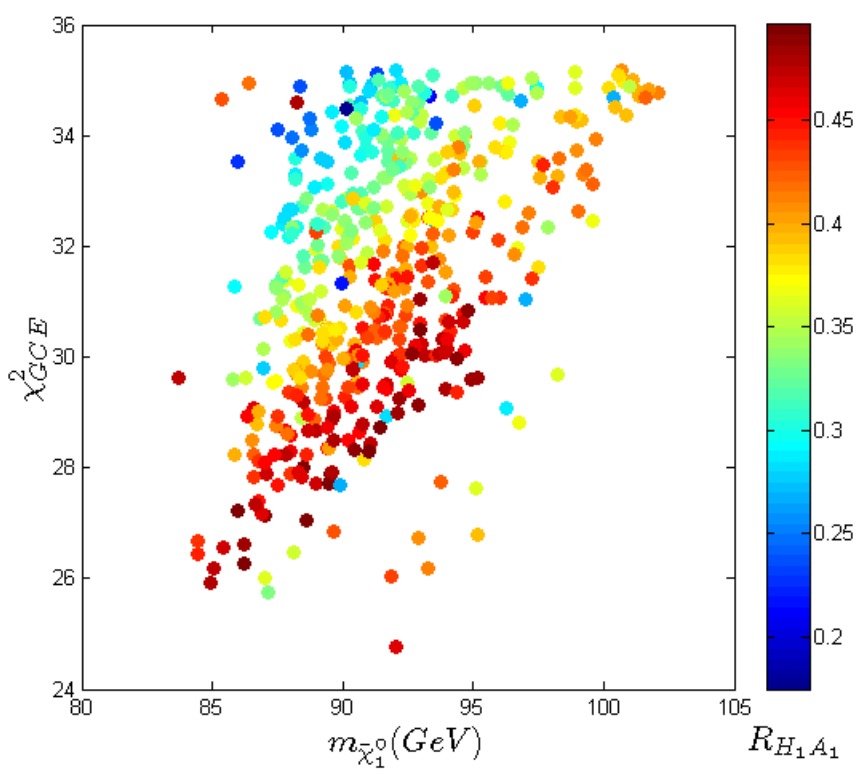

Figure 4. The GCE $\chi^{2}$ as a function of DM mass for Solution III where DM annihilates mainly into $W W$ final state. Here $R_{H_{1} A_{1}}$ denotes the branching ratio of the annihilation into $H_{1} A_{1}$ final state.

Compared with the case that $\mathrm{H}_{2}$ acts as the SM-like Higgs boson, we find that it is more difficult to get the GCE solutions if $H_{1}$ corresponds to the SM-like Higgs boson. One important reason is that the spectrum of the singlet-dominated particles for Solution IV and V has non-trivial requirements on the NMSSM parameters, which can not be easily satisfied due to the structure of the NMSSM itself. A good example about this argument is that we do not find any solutions where DM mainly annihilates into $b \bar{b}$. This is due to the fact that, given a singlino-dominated $\mathrm{DM}$ with $30 \mathrm{GeV} \leq m_{\tilde{\chi}_{1}^{0}} \leq 70 \mathrm{GeV}$ and meanwhile a singlet-dominated $A_{1}$ satisfying $m_{A_{1}} \simeq 2 m_{\tilde{\chi}_{1}^{0}}$, the singlet-dominated CP-even Higgs boson is usually lighter than the SM-like Higgs boson [73].

In table 2, we present detailed information for benchmark points P4, P5, P6 and P7 with points P4 and P5 belonging to Solution IV and points P6 and P7 belonging to Solution $\mathrm{V}$. This table shows that for $80 \mathrm{GeV} \lesssim m_{\tilde{\chi}_{1}^{0}} \lesssim 86 \mathrm{GeV}$, DM may annihilate into $H_{1} A_{1}$ and $H_{2} A_{1}$ states with comparable rates to explain the GCE (see points $\mathrm{P} 5$ and $\mathrm{P} 6$ ), while for $m_{\tilde{\chi}_{1}^{0}} \simeq 78 \mathrm{GeV}\left(m_{\tilde{\chi}_{1}^{0}} \simeq 120 \mathrm{GeV}\right)$, the sole annihilation channel $\tilde{\chi}_{1}^{0} \tilde{\chi}_{1}^{0} \rightarrow H_{1} A_{1}$ $\left(\tilde{\chi}_{1}^{0} \tilde{\chi}_{1}^{0} \rightarrow H_{2} A_{1}\right)$ can be responsible for the GCE, see point P4 (P7). Note that for point P5, the SM-like Higgs boson $H_{1}$ and the singlet-dominated scalar $H_{2}$ are nearly degenerate in mass so that both of them contribute to the signals measured by the ATLAS and CMS collaborations in the discovery of the $125 \mathrm{GeV}$ Higgs boson [135, 136]. This situation was intensively studied in $[137,138]$ in 2012 , and in our work, we have included the two contributions in the Higgs data fit by using the package HiggsSingal [131]. Furthermore, as far as point P5 is concerned, the degeneracy is not easy to discern at the LHC by the double ratios of event rates defined in [138]. The reason is two-fold. One is that $\mathrm{H}_{2}$ is a singlet dominated scalar, so its production rate at the LHC is suppressed. The other is that the dominant signal of $H_{2}$ is $H_{2} \rightarrow A_{1} A_{1} \rightarrow 4 b$, which can be easily overwhelmed in large 


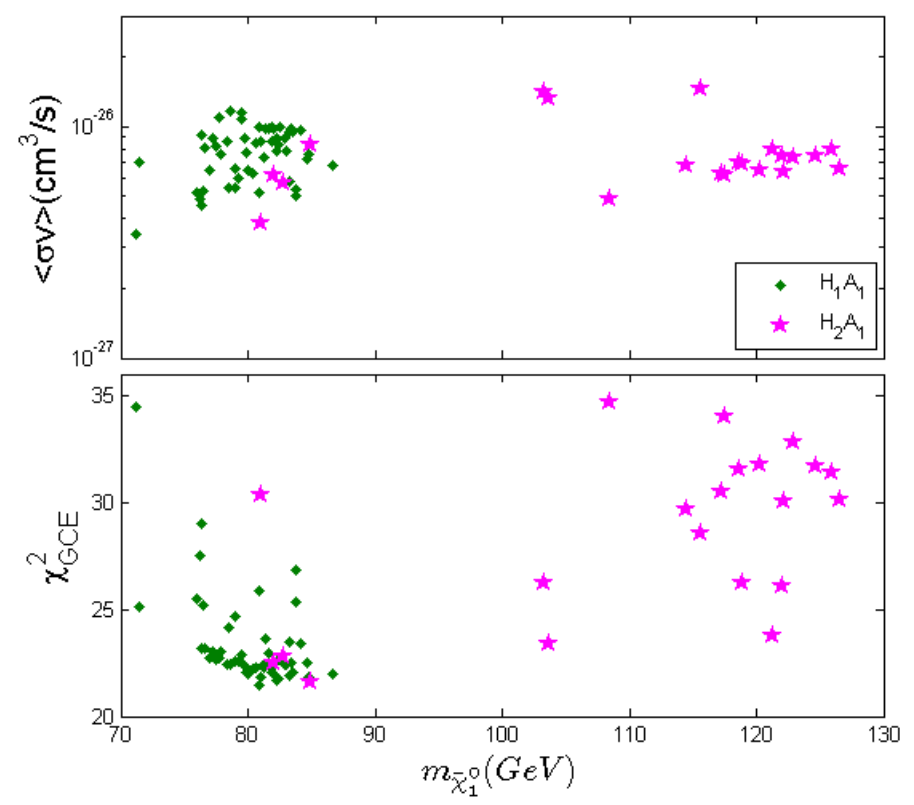

Figure 5. Similar to figure 2, but showing the solutions for the case that $H_{1}$ acts as the SM-like Higgs boson. For these solutions, DM may mainly annihilate into $H_{1} A_{1}$ final state (called Solution IV in our discussion) or into $H_{2} A_{1}$ final state (Solution V).

SM background [112]. Consequently, the contribution of $H_{2}$ to the signals measured by the two collaborations is very small, and the double ratios locate to a good approximation at 1.

In figure 6, we show the correlations among the parameters $\lambda, \kappa, \mu, m_{\tilde{\chi}_{1}^{0}}$ and $m_{A_{1}}$. This figure is supplement to table 1 , and as we will show below, it is helpful for our understanding on Solution IV and V.

\subsection{Solution IV - the $H_{1} A_{1}$ annihilation channel}

Solution IV has the following features:

- The $H_{1} A_{1}$ state must be produced close to threshold to explain the GCE, which is reflected by $\delta<0.1$ from our results.

- The favored spectrum for the singlet-dominated particles is $71 \mathrm{GeV} \lesssim m_{\tilde{\chi}_{1}^{0}} \lesssim 87 \mathrm{GeV}$, $10 \mathrm{GeV} \lesssim m_{A_{1}} \lesssim 40 \mathrm{GeV}$ and $126 \mathrm{GeV} \lesssim m_{H_{2}} \lesssim 142 \mathrm{GeV}$. Given $\kappa \sim 0.12$ which is required to predict the right size of the annihilation $\tilde{\chi}_{1}^{0} \tilde{\chi}_{1}^{0} \rightarrow H_{1} A_{1}$ for the GCE (see below), this spectrum limits parameters such as $\lambda, \kappa, \mu$ and $A_{\kappa}$ within rather narrow ranges, which are given in table 1 and also shown in figure 6 .

Compared with Solution II, we find in Solution IV that, in order to predict a heavier singlet-dominated CP-even Higgs boson, the parameter $\mu$ usually takes a larger value, $220 \mathrm{GeV} \lesssim \mu \lesssim 270 \mathrm{GeV}$. As a result, $\lambda$ must exceed about 0.6 , which can be inferred from the relation $m_{\tilde{\chi}_{1}^{0}} \simeq 2 \kappa \mu / \lambda \simeq 2 \times 0.12 \times \mu / \lambda \simeq 80 \mathrm{GeV}$. This relation also suggests that $v_{s} \equiv \mu / \lambda \gtrsim 300 \mathrm{GeV}$ or $\lambda v / \mu<0.6$, which makes the expansions listed in appendix feasible. 

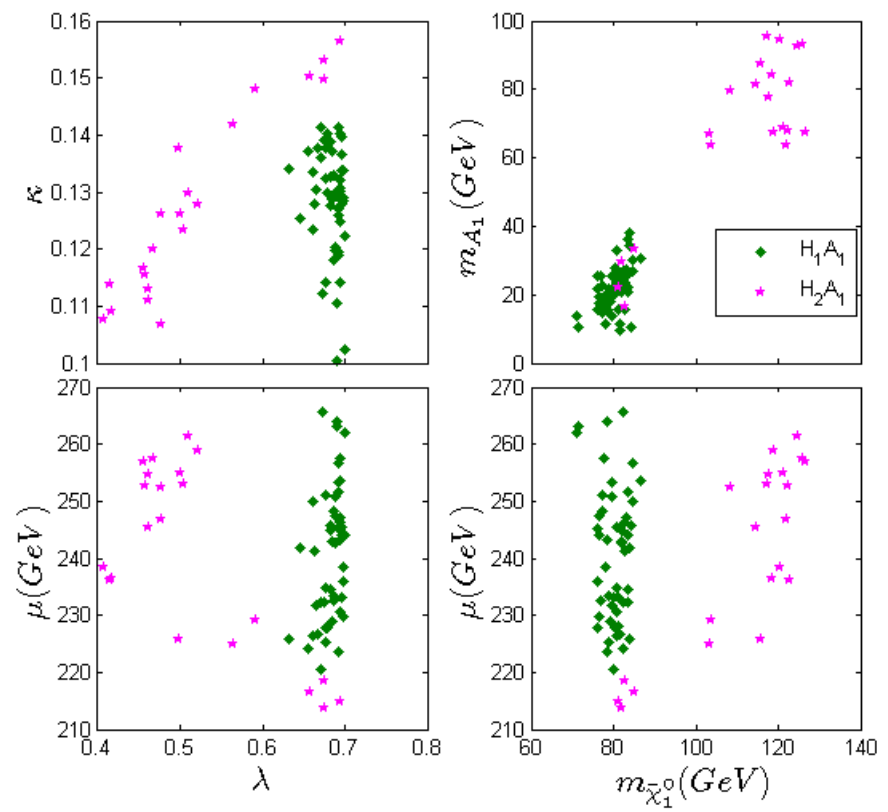

Figure 6. Similar to figure 3, but showing the correlations for Solution IV and Solution V.

- Similar to Solution II, the s-channel contributions to the annihilation rate $\left\langle\sigma_{H_{1} A_{1}} v\right\rangle_{0}$ in eq. (2.5) are usually much smaller than those from the $t / u$ channel, and among the $t / u$ channel contributions, the one induced by the exchange of $\tilde{\chi}_{1}^{0}$ is dominant. However, since $H_{1}$ now is the SM-like Higgs boson (instead of a singlet-dominated particle in Solution II), there still exists a slight difference between the two solutions. Explicitly speaking, we find that each higgsino contribution to the annihilation is comparable in magnitude with the $\tilde{\chi}_{1}^{0}$ contribution, but since the two higgsino contributions cancel each other, the total higgsino contribution is small. These features can be explained by the following formula (see eq. (A.20))

$$
\begin{aligned}
& C_{A_{1} \tilde{\chi}_{1}^{0} \tilde{\chi}_{1}^{0}} C_{H_{1} \tilde{\chi}_{1}^{0} \tilde{\chi}_{1}^{0}} \simeq-4 i \kappa^{2} \frac{\lambda v}{\mu} \sin ^{2} \beta \simeq-8 i \kappa^{3} \frac{v}{m_{\tilde{\chi}_{1}^{0}}} \sin ^{2} \beta, \\
& C_{A_{1} \tilde{\chi}_{1}^{0} \tilde{\chi}_{i}^{0}} C_{H_{1} \tilde{\chi}_{1}^{0} \tilde{\chi}_{i}^{0}} \simeq\left\{\begin{array}{c}
\frac{i}{4} \lambda^{2} \frac{\lambda v}{\mu} \sin ^{2} \beta, \quad \text { for Higgsino }- \text { like } \tilde{\chi}_{i}^{0} \text { and } m_{\tilde{\chi}_{i}^{0}}<0, \\
-\frac{i}{4} \lambda^{2} \frac{\lambda v}{\mu} \sin ^{2} \beta, \quad \text { for Higgsino }- \text { like } \tilde{\chi}_{i}^{0} \text { and } m_{\tilde{\chi}_{i}^{0}}>0,
\end{array}\right.
\end{aligned}
$$

and also by comparing eq. (4.1) with equation (3.20) in [98] to conclude that $\kappa \sim 0.12$ is enough to predict the $\tilde{\chi}_{1}^{0}$ contributed $\left\langle\sigma_{H_{1} A_{1}} v\right\rangle_{0}$ at the order of $10^{-26} \mathrm{~cm}^{3} / \mathrm{s}$.

- Since $m_{A_{1}} \lesssim 40 \mathrm{GeV}$ for all cases in Solution IV (see upper right panel of figure 6 ), the SM-like Higgs boson $H_{1}$ will decay into $A_{1} A_{1}$. We checked that $\operatorname{Br}\left(H_{1} \rightarrow A_{1} A_{1}\right) \lesssim$ $24 \%$ as required by the Higgs data.

- We also checked that $A_{1} \rightarrow b \bar{b}$ is the dominant decay mode of $A_{1}$, and $\operatorname{Br}\left(A_{1} \rightarrow\right.$ $\gamma \gamma)<5 \times 10^{-5}$ for all samples. 


\subsection{Solution $\mathbf{V}$ - the $H_{2} A_{1}$ annihilation channel}

Since $\mathrm{H}_{2}$ in Solution $\mathrm{V}$ is singlet dominated, the features of Solution V should be similar to those of Solution II. The differences mainly come from the following aspects:

- The spectrum of the singlet dominated particles. In Solution $\mathrm{V}$, the favored spectrum is $80 \mathrm{GeV} \lesssim m_{\tilde{\chi}_{1}^{0}} \lesssim 130 \mathrm{GeV}, 18 \mathrm{GeV} \lesssim m_{A_{1}} \lesssim 100 \mathrm{GeV}$ and $125 \mathrm{GeV} \lesssim m_{H_{2}} \lesssim$ $146 \mathrm{GeV}$ with $m_{A_{1}}<m_{\tilde{\chi}_{1}^{0}}<m_{H_{2}}$ and $\delta<0.1$. Corresponding to such a spectrum, the parameter space of Solution V differs greatly from that of Solution II, which can be seen from table 1 and also from figure 6 .

- The phenomenology of some relevant particles. For example, in both Solution IV and Solution $\mathrm{V}$, the favored value of $\mu$ is uplifted in comparison with that in Solution II. As a result, the higgsino-dominated neutralinos may decay into $Z \tilde{\chi}_{1}^{0}$, which makes them to be potentially detected at $14-\mathrm{TeV}$ LHC by trilepton $+E_{T}^{\text {miss }}$ signals [73].

\section{Explore the GCE solutions in future DM experiments}

In this section we investigate to what extent the GCE solutions will be explored in future DM direct detection experiments such as XENON-1T and LUX experiments [139], which will improve current experimental sensitivities to DM-nucleon scattering cross sections by up to three orders. In figure 7 , we project our solutions on $m_{\tilde{\chi}_{1}^{0}}-\sigma_{p}^{\mathrm{SI}}$ and $m_{\tilde{\chi}_{1}^{0}}-\sigma_{p}^{\mathrm{SD}}$ planes with $\sigma_{p}^{\mathrm{SI}}$ and $\sigma_{p}^{\mathrm{SD}}$ denoting the spin-independent (SI) and spin-dependent (SD) cross sections respectively. The left panels in the figure are the results for the case that $\mathrm{H}_{2}$ acts as the SM-like Higgs boson, and the right panels are those for the case that $H_{1}$ corresponds to the SM-like Higgs boson. The dotted lines, solid lines, dashed lines and dash dotted lines are the sensitivities to the cross sections set by the XENON-100, LUX, XENON-1T and LZ experiments respectively. Note that so far the XENON-100 experiment has imposed constraints on both SI and SD cross sections, while the LUX experiment only obtained limits on the SI cross section.

For $\sigma_{p}^{\mathrm{SI}}$ in the $H_{2}$ case, we can see from figure 7 that the future XENON-1T experiment is able to probe a large portion of the GCE solutions, and the LZ experiment can test even more solutions. Anyhow, there still exist some solutions remaining untouched by these future experiments. This conclusion can be understood as follows. In the NMSSM after considering the current experimental constraints on sfermion masses, the main contribution to $\sigma_{p}^{\mathrm{SI}}$ comes from the t-channel process mediated by the CP-even Higgses $H_{1,2}$. In this case, the Wilson coefficient $f_{q_{i}}$ for the operator $\bar{\chi}_{1}^{0} \tilde{\chi}_{1}^{0} \bar{q}_{i} q_{i}$ is given by [140]

$$
f_{q_{i}} \simeq \frac{C_{H_{1} \tilde{\chi}_{1}^{0} \tilde{\chi}_{1}^{0}} C_{H_{1} q_{i} q_{i}}}{2 m_{H_{1}}^{2}}+\frac{C_{H_{2} \tilde{\chi}_{1}^{0} \tilde{\chi}_{1}^{0}} C_{H_{2} q_{i} q_{i}}}{2 m_{H_{2}}^{2}},
$$

where $C_{H_{1} \tilde{\chi}_{1}^{0} \tilde{\chi}_{1}^{0}} \simeq-\sqrt{2} \kappa(1+2 \lambda v / \mu)$ and $C_{H_{2} \tilde{\chi}_{1}^{0} \tilde{\chi}_{1}^{0}} \simeq 2 \sqrt{2} \kappa \lambda v / \mu$, which are given by eq. (A.20) and eq. (A.21) respectively. Then eq. (5.1) indicates that, if $\kappa$ is small or if there exists a strong cancelation between the two terms, $f_{q_{i}}$ or equivalently the SI cross section will be suppressed. We numerically checked that the untouched solutions has either of the two characteristics. 


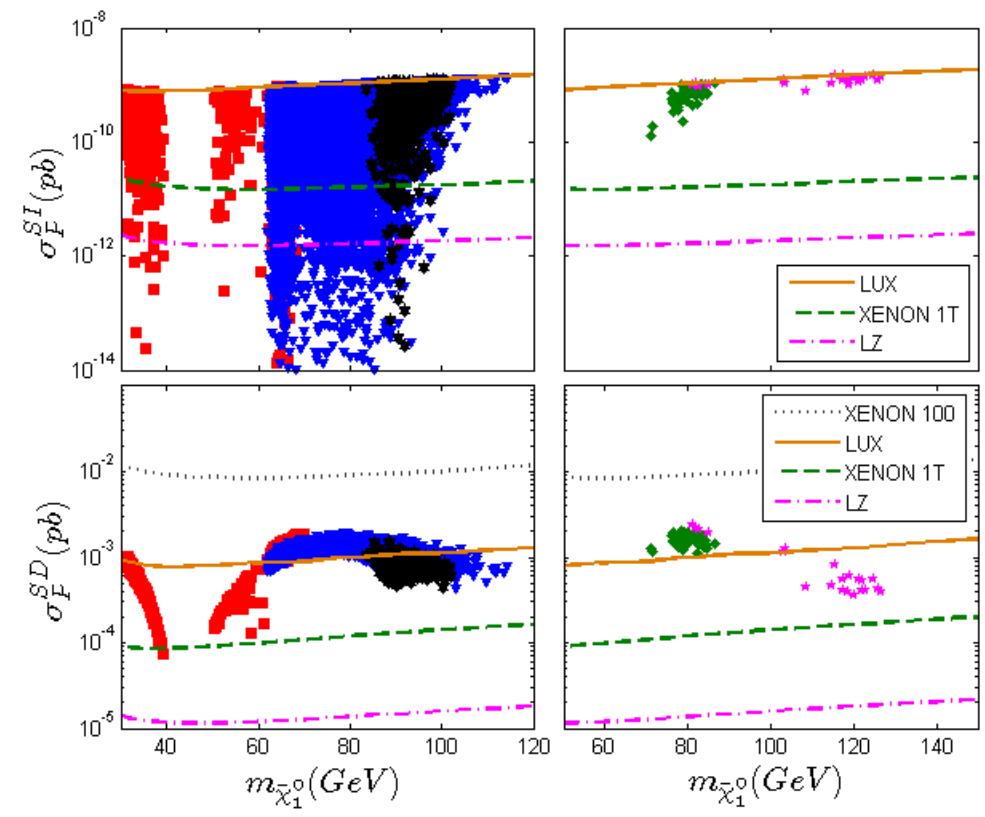

Figure 7. Spin-independent (SI) and Spin-dependent (SD) cross sections for DM-nucleon scattering as a function of DM mass. Solutions in this figure are taken from figure 2 and figure 5 with the same symbolic conventions.

On the other hand, the story for $\sigma_{p}^{\mathrm{SD}}$ in the $H_{2}$ case is quite different. From the lower left panel of figure 7 we can see that the future XENON-1T experiment can test almost all of the GCE solutions, let alone the more sensitive LZ experiment. The underlying reason is that in the NMSSM with heavy sfermions, the SD cross section gets contribution mainly from the $t$-channel $Z$-mediated diagram. As a result, the size of the cross section is determined by the $Z \tilde{\chi}_{1}^{0} \tilde{\chi}_{1}^{0}$ coupling, which is given by

$$
g_{Z \tilde{\chi}_{1}^{0} \tilde{\chi}_{1}^{0}}=\frac{m_{Z}}{\sqrt{2} v}\left(N_{13}^{2}-N_{14}^{2}\right) \simeq-\frac{m_{Z}}{\sqrt{2} v} \frac{\lambda^{2} v^{2}}{\mu^{2}}\left(1-\frac{4 \kappa^{2}}{\lambda^{2}}\right) \simeq-\frac{m_{Z}}{\sqrt{2} v} \frac{\lambda^{2} v^{2}}{\mu^{2}}\left(1-\frac{m_{\tilde{\chi}_{1}^{0}}^{2}}{\mu^{2}}\right) \text {. }
$$

In getting this expression, we have used the approximations for $N_{13}, N_{14}$ and $m_{\tilde{\chi}_{1}^{0}}$. Then from the results presented in figure 3, one can infer that except for some rare cases of Solution I, the SD cross section is not suppressed too much.

In a similar way, one can analyze the results for the $H_{1}$ case, which are shown on the right panels of figure 7. For example, the upper right panel indicates that the SI cross sections in Solution IV and Solution V are usually larger than $10^{-10} p b$. This may be understood by a weak cancelation between the two terms in eq. (5.1). Compared with the $H_{2}$ case, both the SI cross section and the SD cross section in the $H_{1}$ case are large and consequently, all the solutions will be tested by XENON-1T experiment.

In principle, the GCE solutions in the NMSSM may also be tested by electroweakino production processes at the LHC [73]. We will discuss such an issue in our forthcoming work. 


\section{Summary}

In this work, we took into account the recently reported foreground and background uncertainties for the GCE and investigated its explanation by DM annihilation in the framework of the NMSSM. We carried out a sophisticated scan over the NMSSM parameter space by considering various experimental constraints such as the Higgs data, $B$-physics observables, DM relic density, LUX experiment and the dSphs constraints. Then for each surviving parameter point we performed a fit to the GCE spectrum by using the correlation matrix that incorporated both the statistical and systematic uncertainties of the measured excess. Our results indicate that due to the introduction of the gauge singlet Higgs superfield, the NMSSM with a singlino-dominated DM has multiple DM annihilation channels that are able to explain the GCE quite well, and all of these explanations require the singlet-dominated particles (including one neutralino, one CP-even and one CP-odd Higgs bosons) to be moderately light. We also discussed to what extent the future DM direct detection experiments can explore the GCE solutions, and we conclude that the XENON-1T experiment is very promising in testing nearly all the solutions.

When choosing the scenario of particle spectrum, we focused on a singlino-dominated DM and considered the cases that either $H_{2}$ or $H_{1}$ acts as the SM-like Higgs boson. For the popular situation that $\mathrm{H}_{2}$ corresponds to the SM-like Higgs, we have the following observations on the GCE solutions:

- The pure DM annihilation channel $\tilde{\chi}_{1}^{0} \tilde{\chi}_{1}^{0} \rightarrow b \bar{b}$ or $\tilde{\chi}_{1}^{0} \tilde{\chi}_{1}^{0} \rightarrow H_{1} A_{1}$ can provide a good fit to the GCE spectrum, while the channel $\tilde{\chi}_{1}^{0} \tilde{\chi}_{1}^{0} \rightarrow W^{+} W^{-}$must mix sizeably with the channel $\tilde{\chi}_{1}^{0} \tilde{\chi}_{1}^{0} \rightarrow H_{1} A_{1}$ to account for the GCE.

- For the annihilation $\tilde{\chi}_{1}^{0} \tilde{\chi}_{1}^{0} \rightarrow b \bar{b}, \mathrm{DM}$ mass is now allowed in the range from $30 \mathrm{GeV}$ to $70 \mathrm{GeV}$ which is much wider than before. With the help of an appropriate schannel resonance, the singlet trilinear self-coupling parameter $\kappa$ can be as low as 0.02 to explain the GCE. Moreover, the higgsino mass parameter $\mu$ is upper bounded by about $300 \mathrm{GeV}$ to ensure a correct DM relic density. Since there exist strong correlations between independent parameters, such an explanation suffers from a fine tuning problem, which is usually less than $1 \%$.

- The annihilation $\tilde{\chi}_{1}^{0} \tilde{\chi}_{1}^{0} \rightarrow H_{1} A_{1}$ may provide a better explanation than the channel $\tilde{\chi}_{1}^{0} \tilde{\chi}_{1}^{0} \rightarrow b \bar{b}$ when $H_{1} A_{1}$ is produced close to threshold, and the best interpretation corresponds to a p-value of 0.55. In this kind of explanation, the singlet-dominated particles must satisfy $60 \mathrm{GeV} \lesssim m_{\tilde{\chi}_{1}^{0}} \lesssim 115 \mathrm{GeV}, 10 \mathrm{GeV} \lesssim m_{A_{1}} \lesssim 110 \mathrm{GeV}, 60 \mathrm{GeV} \lesssim$ $m_{H_{1}} \lesssim 120 \mathrm{GeV}$ and $\delta<0.2$. This imposes non-trivial constraints on the NMSSM parameters, especially that $\mu$ must be less than about $200 \mathrm{GeV}$. Among various contributions to the annihilation, the dominant one comes from the $\tilde{\chi}_{1}^{0}$-contributed $t / u$ channel diagrams, in which the parameter $\kappa$ plays an important role in deciding the annihilation rate.

- Apart from the necessary mixing with the $H_{1} A_{1}$ final states, $W^{+} W^{-}$pair in the annihilation $\tilde{\chi}_{1}^{0} \tilde{\chi}_{1}^{0} \rightarrow W^{+} W^{-}$must be produced close to threshold to account for the 
GCE. A small $\mu$ less than about $150 \mathrm{GeV}$ is necessary to increase the annihilation rate through the $t / u$-channel contributions induced by a higgsino-dominated chargino. The LHC search for trilepton $+E_{T}^{\text {miss }}$ signal can not exclude such a possibility since the electroweakino production rates at the LHC are relatively low, and meanwhile since the splitting between $\mu$ and $m_{\tilde{\chi}_{1}^{0}}$ is compressed.

- The detection of spin-independent scattering in the future XENON-1T and LUX experiments are able to cover a large portion of the GCE-favored parameter space, while the spin-dependent detection have a stronger potential to test nearly all of the relevant parameter region.

As for the case that $H_{1}$ acts as the SM-like Higgs boson, the features of the GCE solutions are quite different, which are as follows:

- In comparison with the $H_{2}$ case, it is difficult to find GCE solutions when $H_{1}$ corresponds to the SM-like Higgs boson, and especially we did not find any solution that DM annihilates mainly into $b \bar{b}$. The reason is, assuming $H_{1}$ to be the SM-like Higgs boson, there must exist sizeable mass splittings among the light singlet-dominated particles to explain the GCE, which is difficult to realize in the NMSSM due to the theoretical structure itself.

- For $80 \mathrm{GeV} \lesssim m_{\tilde{\chi}_{1}^{0}} \lesssim 86 \mathrm{GeV}, \mathrm{DM}$ may annihilate into $H_{1} A_{1}$ and $H_{2} A_{1}$ states with comparable rates to explain the GCE, while for $m_{\tilde{\chi}_{1}^{0}} \lesssim 80 \mathrm{GeV}\left(m_{\tilde{\chi}_{1}^{0}} \gtrsim 100 \mathrm{GeV}\right)$, the sole annihilation $\tilde{\chi}_{1}^{0} \tilde{\chi}_{1}^{0} \rightarrow H_{1} A_{1}\left(\tilde{\chi}_{1}^{0} \tilde{\chi}_{1}^{0} \rightarrow H_{2} A_{1}\right)$ can be responsible for the GCE. For all these solutions, the singlet-dominated particle $H_{2}$ and the parameter $\mu$ must satisfy $125 \mathrm{GeV} \lesssim m_{H_{2}} \lesssim 145 \mathrm{GeV}$ and $210 \mathrm{GeV} \lesssim \mu \lesssim 270 \mathrm{GeV}$

- Both the spin-independent and spin-dependent detection in the future XENON-1T experiment have a great potential to test the relevant parameter space.

Before we end our discussion, we would like to comment briefly on the interpretation of the GCE with a bino-like DM. Like the singlino-dominated DM case, a light $A_{1}$ with mass below about $140 \mathrm{GeV}$ is necessary for such to work, and this $A_{1}$ prefers to be singletdominated. ${ }^{3}$ The difference is that, for the bino-like DM case, the interaction of the DM with $A_{1}$ is relatively small and consequently the annihilation $\tilde{\chi}_{1}^{0} \tilde{\chi}_{1}^{0} \rightarrow H_{i} A_{1}$ can not explain

\footnotetext{
${ }^{3}$ In the NMSSM, a light $A_{1}$ with mass below about $100 \mathrm{GeV}$ may have a large doublet component if the elements of the CP-odd Higgs mass matrix satisfy $\mathcal{M}_{P, 22}^{2} \gg \mathcal{M}_{P, 12}^{2} \gg \mathcal{M}_{P, 11}^{2}$ (see benchmark points P3 and $\mathrm{P} 4$ in [141]) or $\mathcal{M}_{P, 22}^{2} \simeq \mathcal{M}_{P, 11}^{2} \sim \mathcal{M}_{P, 12}^{2}$ (see the point presented in table 2 of [142]). In either case, $m_{A_{1}}$ should be significantly smaller than $m_{H^{ \pm}}$to escape experimental constraints. Previous studies have suggested that a light doublet-dominated $A_{1}$ might also explain the galactic center excess. However, due to the requirements on the elements this scenario occurs only in specific portions of the parameter space and is significantly more experimentally constrained than those we considered. In fact, in our scans for the GCE we did not find any parameter points with the doublet component of the light $A_{1}$ exceeding 0.1 . In summary, a light doublet-dominated $A_{1}$ may exist, as suggested by e.g. ref. [142], but it is fair to say that without a very delicate parameter tuning, it is difficult to obtain in explaining the GCE, especially when one considers more constraints than previous literatures. About this conclusion, we thank the authors of [142] for helpful discussion.
} 
the GCE any more due to its rather low annihilation rate. Also due to the suppressed interaction, $m_{A_{1}}$ must be closer to $2 m_{\tilde{\chi}_{1}^{0}}$ for the annihilation $\tilde{\chi}_{1}^{0} \tilde{\chi}_{1}^{0} \rightarrow b \bar{b}$ to account for the GCE, and thus the theory has to be tuned in a more elaborated way. Our sophisticated scan over the relevant NMSSM parameter space verified these conclusions.

\section{Acknowledgments}

This work was supported by the National Natural Science Foundation of China (NNSFC) under grant No. 10821504, 11222548, 11121064, 11135003, 90103013 and 11275245, and by the CAS Center for Excellence in Particle Physics (CCEPP).

\section{A Properties of the singlet-dominated particles}

In this appendix, we present some analytic expressions for the masses and couplings of the singlet dominated particles, such as $\tilde{\chi}_{1}^{0}$ and $A_{1}$ in the NMSSM. These expressions are obtained by diagonalizing the mass matrices of the particles (like done in [60]), and are good approximations in certain cases. They are helpful in understanding the results presented in this work. In the following, we will follow notations and conventions consistent with [110] for the $Z_{3}$ NMSSM.

\section{A.1 Neutralino masses and mixings}

In the basis $\psi^{0}=\left(-i \lambda_{1},-i \lambda_{2}^{3}, \psi_{d}^{0}, \psi_{u}^{0}, \psi_{S}\right)$, the neutralino mass matrix is:

$$
\mathcal{M}=\left(\begin{array}{ccccc}
M_{1} & 0 & -\frac{g_{1} v_{d}}{\sqrt{2}} & \frac{g_{1} v_{u}}{\sqrt{2}} & 0 \\
& M_{2} & \frac{g_{2} v_{d}}{\sqrt{2}} & -\frac{g_{2} v_{u}}{\sqrt{2}} & 0 \\
& & 0 & -\mu & -\lambda v_{u} \\
& & & 0 & -\lambda v_{d} \\
& & & & \frac{2 \kappa}{\lambda} \mu
\end{array}\right) .
$$

If the bino and wino fields are decoupled, the mass eigenstates of the neutralinos can be approximated by

$$
\begin{aligned}
& \tilde{\chi}_{1}^{0} \approx N_{13} \psi_{d}^{0}+N_{14} \psi_{u}^{0}+N_{15} \psi_{S} \\
& \tilde{\chi}_{i}^{0} \approx N_{i 3} \psi_{d}^{0}+N_{i 4} \psi_{u}^{0}+N_{i 5} \psi_{S}
\end{aligned}
$$

where $\tilde{\chi}_{1}^{0}$ denotes the lightest neutralino with $\psi_{S}$ field as its dominant component in this work, and $\tilde{\chi}_{i}^{0}$ represents a higgsino-like neutralino. 
In the limit of $|\mu| \gg \lambda v, 1 \gg \kappa / \lambda$ and $\tan \beta \gg 1$, one can expand the neutralino masses and $N_{i j}$ by the power of $\lambda v / \mu \equiv v / v_{s}$ to get the following approximations:

$$
\begin{aligned}
& m_{\tilde{\chi}_{1}^{0}} \approx \frac{2 \kappa}{\lambda} \mu+\frac{\lambda^{2} v^{2}}{\mu^{2}}\left(\mu \sin 2 \beta-\frac{2 \kappa}{\lambda} \mu\right), \\
& \frac{N_{13}}{N_{15}}=\frac{\lambda v}{\mu^{2}-m_{\tilde{\chi}_{1}^{0}}^{2}} \cos \beta\left(\tan \beta m_{\tilde{\chi}_{1}^{0}}-\mu\right) \approx \frac{2 \kappa v}{\mu} \sin \beta, \\
& \frac{N_{14}}{N_{15}}=\frac{-\lambda v}{\mu^{2}-m_{\tilde{\chi}_{1}^{0}}^{2}} \sin \beta\left(\mu-\frac{m_{\tilde{\chi}_{1}^{0}}}{\tan \beta}\right) \approx-\frac{\lambda v}{\mu} \sin \beta, \\
& N_{15}=\left(1+\frac{N_{13}^{2}}{N_{15}^{2}}+\frac{N_{14}^{2}}{N_{15}^{2}}\right)^{-1 / 2} \approx 1, \quad N_{i 3} \approx \frac{1}{\sqrt{2}} \operatorname{Sgn}\left(m_{\tilde{\chi}_{j}^{0}}\right) \theta\left(m_{\tilde{\chi}_{j}^{0}}\right), \\
& N_{i 4} \approx-\frac{1}{\sqrt{2}} \operatorname{Sgn}(\mu) \theta\left(m_{\tilde{\chi}_{j}^{0}}\right), \quad N_{i 5} \approx-\frac{1}{\sqrt{2}} \frac{\lambda v \sin \beta}{\mu} \operatorname{Sgn}(\mu) \theta\left(m_{\tilde{\chi}_{j}^{0}}\right) .
\end{aligned}
$$

In above expressions, the $S g n$ and $\theta$ functions are defined by

$$
\operatorname{Sgn}(x)=\left\{\begin{array}{ll}
1 & \text { if } x \geq 0, \\
-1 & \text { if } x<0,
\end{array} \quad \theta(x)= \begin{cases}1 & \text { if } x \geq 0 \\
i & \text { if } x<0 .\end{cases}\right.
$$

Likewise, one may consider the case that the wino and the singlino fields decouple. In this case, the mass eigenstates of the neutralinos is approximated by

$$
\begin{aligned}
& \tilde{\chi}_{1}^{0} \approx N_{11}\left(-i \lambda_{1}\right)+N_{13} \psi_{d}^{0}+N_{14} \psi_{u}^{0}, \\
& \tilde{\chi}_{i}^{0} \approx N_{i 1}\left(-i \lambda_{1}\right)+N_{i 3} \psi_{d}^{0}+N_{i 4} \psi_{u}^{0} .
\end{aligned}
$$

In the limit of $\tan \beta \gg 1,|\mu| \gg g_{2} v_{u}$ and $|\mu| \gg M_{1}$, we have the following approximations:

$$
\begin{aligned}
& m_{\tilde{\chi}_{1}^{0}} \approx M_{1}-\frac{m_{Z}^{2} \sin ^{2} \theta_{W}}{\mu^{2}}\left(\mu \sin 2 \beta+M_{1}\right), \\
& \frac{N_{13}}{N_{11}} \approx \frac{m_{Z} \sin \theta_{W}}{\mu} \sin \beta \\
& \frac{N_{14}}{N_{11}} \approx-\frac{m_{Z} \sin \theta_{W}}{\mu} \cos \beta\left(1+\tan \beta \frac{M_{1}}{\mu}\right), \\
& N_{11}=\left(1+\frac{N_{13}^{2}}{N_{11}^{2}}+\frac{N_{14}^{2}}{N_{11}^{2}}\right)^{-1 / 2} \approx 1, \quad N_{i 3} \approx \frac{1}{\sqrt{2}} \operatorname{Sgn}\left(m_{\tilde{\chi}_{j}^{0}}\right) \theta\left(m_{\tilde{\chi}_{j}^{0}}\right), \\
& N_{i 4} \approx-\frac{1}{\sqrt{2}} \operatorname{Sgn}(\mu) \theta\left(m_{\tilde{\chi}_{j}^{0}}\right), \quad N_{i 1} \approx \frac{1}{\sqrt{2}} \frac{m_{Z} \sin \theta_{W} \sin \beta}{\mu} \operatorname{Sgn}(\mu) \theta\left(m_{\tilde{\chi}_{j}^{0}}\right) .
\end{aligned}
$$

\section{A.2 CP-odd Higgs mass matrix}

In the $\left(A, S_{I}\right)$ "interaction" basis, the mass matrix for CP-odd Higgs bosons is given by

$$
\mathcal{M}_{P}^{2}=\left(\begin{array}{cc}
m_{A}^{2} & \lambda v\left(\frac{m_{A}^{2}}{2 \mu} \sin 2 \beta-\frac{3 \kappa \mu}{\lambda}\right) \\
& \lambda^{2} v^{2} \sin 2 \beta\left(\frac{m_{A}^{2}}{4 \mu^{2}} \sin 2 \beta+\frac{3 \kappa}{2 \lambda}\right)-\frac{3 \kappa A_{\kappa} \mu}{\lambda}
\end{array}\right) .
$$


In the case of $m_{A} \gg \max \left(v,\left|A_{\kappa}\right|,|\mu|\right), \kappa / \lambda \ll 1$ and $\tan \beta \gg 1$, the lighter CP-odd scalar $A_{1}$ is singlet dominated with its squared mass given by

$$
m_{A_{1}}^{2} \approx \frac{9}{2} \lambda \kappa v^{2} \sin 2 \beta-\frac{3 \kappa A_{\kappa} \mu}{\lambda} .
$$

This approximation indicates that, without considering the radiative corrections, the singlet-dominated CP-odd scalar mass is determined by the parameters $\lambda, \kappa, \mu$ as well as $A_{\kappa}$. The components of $A_{1}$ can be written as

$$
\begin{aligned}
& \frac{P_{A_{1}, A}}{P_{A_{1}, S_{I}}} \approx-\frac{\lambda v}{m_{A}^{2}}\left(\frac{m_{A}^{2}}{2 \mu} \sin 2 \beta-3 \frac{\kappa \mu}{\lambda}\right) \approx-\frac{\lambda v}{2 \mu} \sin 2 \beta, \\
& P_{A_{1}, S_{I}}=\left(1+\frac{P_{A_{1}, A}^{2}}{P_{A_{1}, S_{I}}^{2}}\right)^{-1 / 2} \approx 1,
\end{aligned}
$$

where $P_{A_{1}, A}$ is the active component and $P_{A_{1}, S_{I}}$ is the singlet component of the $A_{1}$.

\section{A.3 CP-even Higgs mass matrix}

In the basis $\left(S_{1}=\cos \beta h_{u}-\sin \beta h_{d}, S_{2}=\sin \beta h_{u}+\cos \beta h_{d}, S_{3}=h_{S}\right)$, the mass matrix elements for the CP-even scalars are [113]

$$
\begin{aligned}
& \mathcal{M}_{11}^{2}=M_{A}^{2}+\left(m_{Z}^{2}-\lambda^{2} v^{2}\right) \sin ^{2} 2 \beta \\
& \mathcal{M}_{12}^{2}=-\frac{1}{2}\left(m_{Z}^{2}-\lambda^{2} v^{2}\right) \sin 4 \beta \\
& \mathcal{M}_{13}^{2}=-\left(M_{A}^{2} \sin 2 \beta+\frac{2 \kappa \mu^{2}}{\lambda}\right) \frac{\lambda v}{\mu} \cos 2 \beta \\
& \mathcal{M}_{22}^{2}=m_{Z}^{2} \cos ^{2} 2 \beta+\lambda^{2} v^{2} \sin ^{2} 2 \beta \\
& \mathcal{M}_{23}^{2}=2 \lambda \mu v\left[1-\left(\frac{M_{A} \sin 2 \beta}{2 \mu}\right)^{2}-\frac{\kappa}{2 \lambda} \sin 2 \beta\right], \\
& \mathcal{M}_{33}^{2}=\frac{1}{4} \lambda^{2} v^{2}\left(\frac{M_{A} \sin 2 \beta}{\mu}\right)^{2}+\frac{\kappa \mu}{\lambda}\left(A_{\kappa}+\frac{4 \kappa \mu}{\lambda}\right)-\frac{1}{2} \lambda \kappa v^{2} \sin 2 \beta,
\end{aligned}
$$

where $S_{2}$ is nothing but the Higgs field in the $\mathrm{SM}, \mathcal{M}_{22}^{2}$ is its mass at tree level without considering the mixing among $S_{i}$, and the second term $\lambda^{2} v^{2} \sin ^{2} 2 \beta$ in $\mathcal{M}_{22}^{2}$ originates from the coupling $\lambda \hat{H}_{u} \cdot \hat{H}_{d} \hat{S}$ in the superpotential.

The mass eigenstates $H_{i}$ are defined by

$$
H_{i}=V_{i 1} S_{1}+V_{i 2} S_{2}+V_{i 3} S_{3}
$$

where $V$ is the rotation matrix to diagonalize the mass matrix. For the $S_{2}$-dominated mass eigenstate $H_{j}$, current Higgs data have required it to be highly SM-like, i.e. $V_{j, 1}, V_{j, 3} \ll 1$, so in the case of the hierarchy structure $\mathcal{M}_{11}^{2} \gg \max \left(\mathcal{M}_{22}^{2}, \mathcal{M}_{33}^{2}\right), \mathcal{M}_{23}^{2} \ll\left|\mathcal{M}_{22}^{2}-\mathcal{M}_{33}^{2}\right|$. If we decouple the MSSM-like heavy Higgs, $S_{1}$, from the other two, the $2 \times 2$ reduced mass matrix in the $\left(S_{2}, S_{3}\right)$ basis is given by [113]:

$$
\mathcal{M}_{S_{2} S_{3}}^{2}=\left(\begin{array}{cc}
\mathcal{M}_{22}^{2} & \mathcal{M}_{23}^{2} \\
& \mathcal{M}_{33}^{2}-\frac{\lambda^{2} v^{2} m_{A}^{2}}{16 \mu^{2}} \sin ^{2} 4 \beta-\frac{\kappa^{2} \mu^{2} v^{2}}{m_{A}^{2}} \cos ^{2} 2 \beta-\lambda \kappa v^{2} \cos ^{2} 2 \beta \sin 2 \beta
\end{array}\right)
$$


The $(2,2)$ element of the reduced $(2 \times 2)$ matrix, which in the limit of zero-mixing with the other Higgs should give singlet scalar mass in the $Z_{3}$ NMSSM, is given by:

$$
\begin{aligned}
\mathcal{M}_{S_{2} S_{3}}^{2}(2,2)= & \frac{\kappa \mu}{\lambda}\left(A_{\kappa}+\frac{4 \kappa \mu}{\lambda}\right)+\frac{\lambda^{2} v^{2} m_{A}^{2}}{4 \mu^{2}}\left(1-\cos ^{2} 2 \beta\right) \sin ^{2} 2 \beta \\
& -\frac{\kappa^{2} \mu^{2} v^{2}}{m_{A}^{2}} \cos ^{2} 2 \beta-\frac{1}{2} \kappa \lambda v^{2}\left(2 \cos ^{2} 2 \beta+1\right) \sin 2 \beta .
\end{aligned}
$$

Setting $\mathcal{M}_{23}^{2} \sim 0$, i.e. $m_{A}^{2}=\frac{4 \mu^{2}}{\sin ^{2} 2 \beta}\left(1-\frac{\kappa}{2 \lambda} \sin 2 \beta\right)$, and taking $\tan \beta \gg 1$, we have

$$
\mathcal{M}_{S_{2} S_{3}}^{2}(2,2) \approx \frac{\kappa \mu}{\lambda}\left(A_{\kappa}+\frac{4 \kappa \mu}{\lambda}\right)
$$

This approximation indicates again that, without considering the radiative corrections, the singlet-dominated CP-even scalar mass is determined by the parameters $\lambda, \kappa, \mu$ and $A_{\kappa}$.

\section{A.4 Some properties of the singlet-dominated particles}

With the assumptions that $M_{A} \gg \max \left(|\mu|,\left|A_{\kappa}\right|\right),|\mu| \gg \lambda v, \tan \beta \gg 1$ and $\kappa / \lambda \ll 1$, one can approximate the masses and couplings of the singlet dominated particles, such as $\tilde{\chi}_{1}^{0}$ and $A_{1}$, by simple analytic expressions [60]. In the following, we list some of the coupling expressions used in our discussion, which are denoted by $C_{X Y Z}$ hereafter. These expressions are actually expand the corresponding exact ones by the power of $\lambda v / \mu$.

$$
\begin{aligned}
& C_{A_{1} b \bar{b}}=\frac{i m_{b} \tan \beta}{\sqrt{2} v} P_{A_{1} A} \approx-\frac{i m_{b}}{\sqrt{2} v} \frac{\lambda v}{\mu}, \\
& C_{A_{1} \tilde{\chi}_{1}^{0} \tilde{\chi}_{j}^{0}} \approx \begin{cases}-i \sqrt{2} \kappa\left(1+2 \frac{\lambda v}{\mu}\right) & \text { for } j=1, \\
-\frac{i \lambda}{2} \frac{\lambda v}{\mu} \operatorname{Sgn}\left(m_{\tilde{\chi}_{j}^{0}}\right) \theta\left(m_{\tilde{\chi}_{j}^{0}}\right) & \text { for higgsino-like } \tilde{\chi}_{j}^{0},\end{cases} \\
& C_{H_{i} \tilde{\chi}_{1}^{0} \tilde{\chi}_{j}^{0}}=-i C_{A_{1} \tilde{\chi}_{1}^{0} \tilde{\chi}_{j}^{0}} \quad \text { if } H_{i} \text { is singlet dominated. }
\end{aligned}
$$

Likewise, if $H_{i}$ is the SM-like Higgs boson, we have

$$
C_{H_{i} \tilde{\chi}_{1}^{0} \tilde{\chi}_{j}^{0}} \approx \begin{cases}2 \sqrt{2} \kappa \frac{\lambda v}{\mu} & \text { for } j=1, \\ \frac{\lambda}{2} \operatorname{Sgn}\left(m_{\tilde{\chi}_{j}^{0}}\right) \theta\left(m_{\tilde{\chi}_{j}^{0}}\right) & \text { for higgsino - like } \tilde{\chi}_{j}^{0},\end{cases}
$$

Open Access. This article is distributed under the terms of the Creative Commons Attribution License (CC-BY 4.0), which permits any use, distribution and reproduction in any medium, provided the original author(s) and source are credited.

\section{References}

[1] L. Goodenough and D. Hooper, Possible Evidence For Dark Matter Annihilation In The Inner Milky Way From The Fermi Gamma Ray Space Telescope, arXiv:0910.2998 [INSPIRE].

[2] D. Hooper and L. Goodenough, Dark Matter Annihilation in The Galactic Center As Seen by the Fermi Gamma Ray Space Telescope, Phys. Lett. B 697 (2014) 412 [arXiv:1010.2752] [INSPIRE]. 
[3] D. Hooper and T. Linden, On The Origin Of The Gamma Rays From The Galactic Center, Phys. Rev. D 84 (2011) 123005 [arXiv:1110.0006] [InSPIRE].

[4] K.N. Abazajian and M. Kaplinghat, Detection of a Gamma-Ray Source in the Galactic Center Consistent with Extended Emission from Dark Matter Annihilation and Concentrated Astrophysical Emission, Phys. Rev. D 86 (2012) 083511 [Erratum ibid. D 87 (2013) 129902] [arXiv: 1207.6047] [INSPIRE].

[5] C. Gordon and O. Macias, Dark Matter and Pulsar Model Constraints from Galactic Center Fermi-LAT Gamma Ray Observations, Phys. Rev. D 88 (2013) 083521 [arXiv:1306.5725] [INSPIRE].

[6] K.N. Abazajian, N. Canac, S. Horiuchi and M. Kaplinghat, Astrophysical and Dark Matter Interpretations of Extended Gamma-Ray Emission from the Galactic Center, Phys. Rev. D 90 (2014) 023526 [arXiv:1402.4090] [INSPIRE].

[7] D. Hooper and T.R. Slatyer, Two Emission Mechanisms in the Fermi Bubbles: A Possible Signal of Annihilating Dark Matter, Phys. Dark Univ. 2 (2013) 118 [arXiv:1302.6589] [INSPIRE].

[8] T. Daylan et al., The Characterization of the Gamma-Ray Signal from the Central Milky Way: A Compelling Case for Annihilating Dark Matter, arXiv:1402.6703 [INSPIRE].

[9] F. Calore, I. Cholis and C. Weniger, Background model systematics for the Fermi GeV excess, JCAP 03 (2015) 038 [arXiv:1409.0042] [InSPIRE].

[10] S. Murgia, Observation of the high energy gamma-ray emission towards the Galactic center, talk given on Fifth Fermi Symposium, Nagoya, Gapan, 20-24 October 2014.

[11] Q. Yuan and B. Zhang, Millisecond pulsar interpretation of the Galactic center gamma-ray excess, JHEAp 3-4 (2014) 1 [arXiv:1404.2318] [INSPIRE].

[12] I. Cholis, D. Hooper and T. Linden, Challenges in Explaining the Galactic Center Gamma-Ray Excess with Millisecond Pulsars, JCAP 06 (2015) 043 [arXiv:1407.5625] [INSPIRE].

[13] R.M. O'Leary, M.D. Kistler, M. Kerr and J. Dexter, Young Pulsars and the Galactic Center GeV Gamma-ray Excess, arXiv:1504.02477 [INSPIRE].

[14] E. Carlson and S. Profumo, Cosmic Ray Protons in the Inner Galaxy and the Galactic Center Gamma-Ray Excess, Phys. Rev. D 90 (2014) 023015 [arXiv:1405.7685] [inSPIRE].

[15] J. Petrović, P.D. Serpico and G. Zaharijas, Galactic Center gamma-ray "excess" from an active past of the Galactic Centre?, JCAP 10 (2014) 052 [arXiv:1405.7928] [INSPIRE].

[16] B.D. Fields, S.L. Shapiro and J. Shelton, Galactic Center Gamma-Ray Excess from Dark Matter Annihilation: Is There A Black Hole Spike?, Phys. Rev. Lett. 113 (2014) 151302 [arXiv: 1406.4856] [INSPIRE].

[17] B. Zhou et al., GeV excess in the Milky Way: The role of diffuse galactic gamma-ray emission templates, Phys. Rev. D 91 (2015) 123010 [arXiv:1406.6948] [InSPIRE].

[18] C. Gordon and O. Macias, Can Cosmic Rays Interacting With Molecular Clouds Explain The Galactic Center Gamma-Ray Excess?, PoS (CRISM2014) 042 [arXiv:1410.7840] [INSPIRE].

[19] R. Bartels, S. Krishnamurthy and C. Weniger, Strong support for the millisecond pulsar origin of the Galactic center GeV excess, arXiv:1506.05104 [INSPIRE].

[20] I. Cholis, C. Evoli, F. Calore, T. Linden, C. Weniger and D. Hooper, The Galactic Center GeV Excess from a Series of Leptonic Cosmic-Ray Outbursts, arXiv:1506.05119 [INSPIRE]. 
[21] Fermi-LAT collaboration, M. Ackermann et al., Dark matter constraints from observations of 25 Milky Way satellite galaxies with the Fermi Large Area Telescope, Phys. Rev. D 89 (2014) 042001 [arXiv:1310.0828] [INSPIRE].

[22] A. Geringer-Sameth, S.M. Koushiappas and M.G. Walker, Comprehensive search for dark matter annihilation in dwarf galaxies, Phys. Rev. D 91 (2015) 083535 [arXiv:1410.2242] [INSPIRE].

[23] Fermi-LAT collaboration, M. Wood, New Fermi-LAT Results on the Search for Dark Matter Annihilation in Dwarf Spheroidal Galaxies, talk given at SLAC Experimental Physics Seminar, Menlo Park, U.S.A., 14 October 2014.

[24] Fermi-LAT collaboration, B. Anderson, Using Likelihood for Combined Data Set Analysis, talk given at the Fifth Fermi Symposium, Nagoya, Japan, 20-24 October 2014.

[25] Fermi-LAT collaboration, M. Ackermann et al., Searching for Dark Matter Annihilation from Milky Way Dwarf Spheroidal Galaxies with Six Years of Fermi-LAT Data, arXiv: 1503.02641 [INSPIRE].

[26] L. Bergstrom, T. Bringmann, I. Cholis, D. Hooper and C. Weniger, New limits on dark matter annihilation from AMS cosmic ray positron data, Phys. Rev. Lett. 111 (2013) 171101 [arXiv: 1306.3983] [INSPIRE].

[27] A. Ibarra, A.S. Lamperstorfer and J. Silk, Dark matter annihilations and decays after the AMS-02 positron measurements, Phys. Rev. D 89 (2014) 063539 [arXiv:1309.2570] [INSPIRE].

[28] K. Kong and J.-C. Park, Bounds on dark matter interpretation of Fermi-LAT GeV excess, Nucl. Phys. B 888 (2014) 154 [arXiv:1404.3741] [INSPIRE].

[29] Q. Yuan and X.-J. Bi, Systematic study of the uncertainties in fitting the cosmic positron data by AMS-02, JCAP 03 (2015) 033 [arXiv:1408.2424] [INSPIRE].

[30] R. Kappl, A. Reinert and M.W. Winkler, AMS-02 Antiprotons Reloaded, arXiv:1506.04145 [INSPIRE].

[31] T. Bringmann, M. Vollmann and C. Weniger, Updated cosmic-ray and radio constraints on light dark matter: Implications for the GeV gamma-ray excess at the Galactic center, Phys. Rev. D 90 (2014) 123001 [arXiv:1406.6027] [INSPIRE].

[32] M. Cirelli, D. Gaggero, G. Giesen, M. Taoso and A. Urbano, Antiproton constraints on the GeV gamma-ray excess: a comprehensive analysis, JCAP 12 (2014) 045 [arXiv:1407.2173] [INSPIRE].

[33] C. Evoli, I. Cholis, D. Grasso, L. Maccione and P. Ullio, Antiprotons from dark matter annihilation in the Galaxy: astrophysical uncertainties, Phys. Rev. D 85 (2012) 123511 [arXiv: 1108.0664] [INSPIRE].

[34] I. Cholis, New Constraints from PAMELA anti-proton data on Annihilating and Decaying Dark Matter, JCAP 09 (2011) 007 [arXiv:1007.1160] [INSPIRE].

[35] F. Donato, D. Maurin, P. Brun, T. Delahaye and P. Salati, Constraints on WIMP Dark Matter from the High Energy PAMELA $\bar{p} / p$ data, Phys. Rev. Lett. 102 (2009) 071301 [arXiv:0810.5292] [INSPIRE].

[36] D. Hooper, T. Linden and P. Mertsch, What Does The PAMELA Antiproton Spectrum Tell Us About Dark Matter?, JCAP 03 (2015) 021 [arXiv: 1410.1527] [INSPIRE].

[37] B. Kyae and J.-C. Park, Light dark matter for Fermi-LAT and CDMS observations, Phys. Lett. B 732 (2014) 373 [arXiv: 1310.2284] [INSPIRE]. 
[38] K.P. Modak, D. Majumdar and S. Rakshit, A Possible Explanation of Low Energy $\gamma$-ray Excess from Galactic Centre and Fermi Bubble by a Dark Matter Model with Two Real Scalars, JCAP 03 (2015) 011 [arXiv:1312.7488] [INSPIRE].

[39] C. Boehm, M.J. Dolan, C. McCabe, M. Spannowsky and C.J. Wallace, Extended gamma-ray emission from Coy Dark Matter, JCAP 05 (2014) 009 [arXiv:1401.6458] [INSPIRE].

[40] E. Hardy, R. Lasenby and J. Unwin, Annihilation Signals from Asymmetric Dark Matter, JHEP 07 (2014) 049 [arXiv: 1402 .4500] [INSPIRE].

[41] T. Lacroix, C. Boehm and J. Silk, Fitting the Fermi-LAT GeV excess: On the importance of including the propagation of electrons from dark matter, Phys. Rev. D 90 (2014) 043508 [arXiv: 1403.1987] [INSPIRE].

[42] A. Alves, S. Profumo, F.S. Queiroz and W. Shepherd, Effective field theory approach to the Galactic Center gamma-ray excess, Phys. Rev. D 90 (2014) 115003 [arXiv:1403.5027] [INSPIRE].

[43] A. Berlin, D. Hooper and S.D. McDermott, Simplified Dark Matter Models for the Galactic Center Gamma-Ray Excess, Phys. Rev. D 89 (2014) 115022 [arXiv:1404.0022] [InSPIRE].

[44] P. Agrawal, B. Batell, D. Hooper and T. Lin, Flavored Dark Matter and the Galactic Center Gamma-Ray Excess, Phys. Rev. D 90 (2014) 063512 [arXiv:1404.1373] [InSPIRE].

[45] E. Izaguirre, G. Krnjaic and B. Shuve, The Galactic Center Excess from the Bottom Up, Phys. Rev. D 90 (2014) 055002 [arXiv:1404.2018] [INSPIRE].

[46] D.G. Cerdeño, M. Peiró and S. Robles, Low-mass right-handed sneutrino dark matter: SuperCDMS and LUX constraints and the Galactic Centre gamma-ray excess, JCAP 08 (2014) 005 [arXiv: 1404.2572] [INSPIRE].

[47] S. Ipek, D. McKeen and A.E. Nelson, A Renormalizable Model for the Galactic Center Gamma Ray Excess from Dark Matter Annihilation, Phys. Rev. D 90 (2014) 055021 [arXiv: 1404.3716] [INSPIRE].

[48] P. Ko, W.-I. Park and Y. Tang, Higgs portal vector dark matter for $\mathrm{GeV}$ scale $\gamma$-ray excess from galactic center, JCAP 09 (2014) 013 [arXiv: 1404.5257] [INSPIRE].

[49] C. Boehm, M.J. Dolan and C. McCabe, A weighty interpretation of the Galactic Centre excess, Phys. Rev. D 90 (2014) 023531 [arXiv: 1404.4977] [inSPIRE].

[50] M. Abdullah, A. DiFranzo, A. Rajaraman, T.M.P. Tait, P. Tanedo and A.M. Wijangco, Hidden on-shell mediators for the Galactic Center $\gamma$-ray excess, Phys. Rev. D 90 (2014) 035004 [arXiv: 1404.6528] [inSPIRE].

[51] D.K. Ghosh, S. Mondal and I. Saha, Confronting the Galactic Center Gamma Ray Excess With a Light Scalar Dark Matter, JCAP 02 (2015) 035 [arXiv: 1405. 0206] [InSPIRE].

[52] A. Martin, J. Shelton and J. Unwin, Fitting the Galactic Center Gamma-Ray Excess with Cascade Annihilations, Phys. Rev. D 90 (2014) 103513 [arXiv:1405.0272] [InSPIRE].

[53] A. Berlin, P. Gratia, D. Hooper and S.D. McDermott, Hidden Sector Dark Matter Models for the Galactic Center Gamma-Ray Excess, Phys. Rev. D 90 (2014) 015032 [arXiv:1405.5204] [INSPIRE].

[54] T. Mondal and T. Basak, Class of Higgs-portal Dark Matter models in the light of gamma-ray excess from Galactic center, Phys. Lett. B 744 (2015) 208 [arXiv:1405.4877] [INSPIRE].

[55] J.M. Cline, G. Dupuis, Z. Liu and W. Xue, The windows for kinetically mixed Z'-mediated dark matter and the galactic center gamma ray excess, JHEP 08 (2014) 131 [arXiv: 1405.7691] [INSPIRE]. 
[56] T. Han, Z. Liu and S. Su, Light Neutralino Dark Matter: Direct/Indirect Detection and Collider Searches, JHEP 08 (2014) 093 [arXiv: 1406.1181] [INSPIRE].

[57] L. Wang and X.-F. Han, A simplified 2HDM with a scalar dark matter and the galactic center gamma-ray excess, Phys. Lett. B 739 (2014) 416 [arXiv:1406.3598] [INSPIRE].

[58] W.-F. Chang and J.N. Ng, Minimal model of Majoronic dark radiation and dark matter, Phys. Rev. D 90 (2014) 065034 [arXiv: 1406.4601] [INSPIRE].

[59] C. Arina, E. Del Nobile and P. Panci, Dark Matter with Pseudoscalar-Mediated Interactions Explains the DAMA Signal and the Galactic Center Excess, Phys. Rev. Lett. 114 (2015) 011301 [arXiv: 1406 . 5542] [INSPIRE].

[60] C. Cheung, M. Papucci, D. Sanford, N.R. Shah and K.M. Zurek, NMSSM Interpretation of the Galactic Center Excess, Phys. Rev. D 90 (2014) 075011 [arXiv:1406.6372] [InSPIRE].

[61] J. Huang, T. Liu, L.-T. Wang and F. Yu, Supersymmetric subelectroweak scale dark matter, the Galactic Center gamma-ray excess and exotic decays of the 125 GeV Higgs boson, Phys. Rev. D 90 (2014) 115006 [arXiv: 1407.0038] [INSPIRE].

[62] C. Balázs and T. Li, Simplified Dark Matter Models Confront the Gamma Ray Excess, Phys. Rev. D 90 (2014) 055026 [arXiv: 1407.0174] [INSPIRE].

[63] P. Ko and Y. Tang, Galactic center $\gamma$-ray excess in hidden sector DM models with dark gauge symmetries: local $Z_{3}$ symmetry as an example, JCAP 01 (2015) 023 [arXiv:1407.5492] [INSPIRE].

[64] S. Baek, P. Ko and W.-I. Park, Local $Z_{2}$ scalar dark matter model confronting galactic GeV-scale $\gamma$-ray, Phys. Lett. B 747 (2015) 255 [arXiv:1407.6588] [inSPIRE].

[65] N. Okada and O. Seto, Galactic Center gamma-ray excess from two-Higgs-doublet-portal dark matter, Phys. Rev. D 90 (2014) 083523 [arXiv: 1408.2583] [INSPIRE].

[66] K. Ghorbani, Fermionic dark matter with pseudo-scalar Yukawa interaction, JCAP 01 (2015) 015 [arXiv: 1408.4929] [INSPIRE].

[67] N.F. Bell, S. Horiuchi and I.M. Shoemaker, Annihilating Asymmetric Dark Matter, Phys. Rev. D 91 (2015) 023505 [arXiv: 1408.5142] [INSPIRE].

[68] A.D. Banik and D. Majumdar, Low Energy Gamma Ray Excess Confronting a Singlet Scalar Extended Inert Doublet Dark Matter Model, Phys. Lett. B 743 (2015) 420 [arXiv: 1408.5795] [INSPIRE].

[69] D. Borah and A. Dasgupta, Galactic Center Gamma Ray Excess in a Radiative Neutrino Mass Model, Phys. Lett. B 741 (2015) 103 [arXiv:1409.1406] [INSPIRE].

[70] M. Cahill-Rowley, J. Gainer, J. Hewett and T. Rizzo, Towards a Supersymmetric Description of the Fermi Galactic Center Excess, JHEP 02 (2015) 057 [arXiv: 1409.1573] [INSPIRE].

[71] J.-H. Yu, Vector Fermion-Portal Dark Matter: Direct Detection and Galactic Center Gamma-Ray Excess, Phys. Rev. D 90 (2014) 095010 [arXiv: 1409. 3227] [InSPIRE].

[72] J. Guo, J. Li, T. Li and A.G. Williams, NMSSM explanations of the Galactic center gamma ray excess and promising LHC searches, Phys. Rev. D 91 (2015) 095003 [arXiv:1409.7864] [INSPIRE].

[73] J. Cao, L. Shang, P. Wu, J.M. Yang and Y. Zhang, Supersymmetry explanation of the Fermi Galactic Center excess and its test at LHC run II, Phys. Rev. D 91 (2015) 055005 [arXiv: 1410.3239] [INSPIRE]. 
[74] Z.-H. Yu, X.-J. Bi, Q.-S. Yan and P.-F. Yin, Tau Portal Dark Matter models at the LHC, Phys. Rev. D 91 (2015) 035008 [arXiv:1410.3347] [InSPIRE].

[75] M. Freytsis, D.J. Robinson and Y. Tsai, Galactic Center Gamma-Ray Excess through a Dark Shower, Phys. Rev. D 91 (2015) 035028 [arXiv:1410.3818] [InSPIRE].

[76] M. Heikinheimo and C. Spethmann, Galactic Centre GeV Photons from Dark Technicolor, JHEP 12 (2014) 084 [arXiv: 1410.4842] [INSPIRE].

[77] P. Agrawal, B. Batell, P.J. Fox and R. Harnik, WIMPs at the Galactic Center, JCAP 05 (2015) 011 [arXiv: 1411.2592] [INSPIRE].

[78] K. Cheung, W.-C. Huang and Y.-L.S. Tsai, Non-abelian Dark Matter Solutions for Galactic Gamma-ray Excess and Perseus 3.5 keV X-ray Line, JCAP 05 (2015) 053 [arXiv: 1411.2619] [INSPIRE].

[79] J. Petrović, P.D. Serpico and G. Zaharijas, Millisecond pulsars and the Galactic Center gamma-ray excess: the importance of luminosity function and secondary emission, JCAP 02 (2015) 023 [arXiv: 1411.2980] [INSPIRE].

[80] G. Arcadi, Y. Mambrini and F. Richard, Z-portal dark matter, JCAP 03 (2015) 018 [arXiv: 1411.2985] [INSPIRE].

[81] D. Hooper, $Z^{\prime}$ mediated dark matter models for the Galactic Center gamma-ray excess, Phys. Rev. D 91 (2015) 035025 [arXiv: 1411.4079] [INSPIRE].

[82] Q. Yuan and K. Ioka, Testing the millisecond pulsar scenario of the Galactic center gamma-ray excess with very high energy gamma-rays, Astrophys. J. 802 (2015) 124 [arXiv: 1411.4363] [INSPIRE].

[83] F. Calore, I. Cholis, C. McCabe and C. Weniger, A Tale of Tails: Dark Matter Interpretations of the Fermi GeV Excess in Light of Background Model Systematics, Phys. Rev. D 91 (2015) 063003 [arXiv: 1411.4647] [INSPIRE].

[84] J. Liu, N. Weiner and W. Xue, Signals of a Light Dark Force in the Galactic Center, JHEP 08 (2015) 050 [arXiv: 1412.1485] [INSPIRE].

[85] A. Biswas, Explaining Low Energy $\gamma$-ray Excess from the Galactic Centre using a Two Component Dark Matter Model, arXiv:1412.1663 [INSPIRE].

[86] M.J. Dolan, F. Kahlhoefer, C. McCabe and K. Schmidt-Hoberg, A taste of dark matter: Flavour constraints on pseudoscalar mediators, JHEP 03 (2015) 171 [Erratum ibid. 1507 (2015) 103] [arXiv: 1412.5174] [INSPIRE].

[87] K. Ghorbani and H. Ghorbani, Scalar Split WIMPs and Galactic Gamma-Ray Excess, arXiv:1501.00206 [INSPIRE].

[88] D.G. Cerdeno, M. Peiro and S. Robles, Fits to the Fermi-LAT GeV excess with RH sneutrino dark matter: implications for direct and indirect dark matter searches and the LHC, Phys. Rev. D 91 (2015) 123530 [arXiv:1501.01296] [INSPIRE].

[89] A. Biswas, D. Majumdar and P. Roy, Nonthermal two component dark matter model for Fermi-LAT $\gamma$-ray excess and $3.55 \mathrm{keV} X$-ray line, JHEP 04 (2015) 065 [arXiv:1501.02666] [INSPIRE].

[90] A. Alves, A. Berlin, S. Profumo and F.S. Queiroz, Dark Matter Complementarity and the $Z^{\prime}$ Portal, arXiv:1501.03490 [INSPIRE].

[91] M. Kaplinghat, T. Linden and H.-B. Yu, Galactic Center Excess in $\gamma$ Rays from Annihilation of Self-Interacting Dark Matter, Phys. Rev. Lett. 114 (2015) 211303 [arXiv: 1501.03507] [INSPIRE]. 
[92] A. Berlin, A. DiFranzo and D. Hooper, $3.55 \mathrm{keV}$ line from exciting dark matter without a hidden sector, Phys. Rev. D 91 (2015) 075018 [arXiv:1501.03496] [INSPIRE].

[93] C.-H. Chen and T. Nomura, $\mathrm{SU}(2)_{X}$ vector DM and Galactic Center gamma-ray excess, Phys. Lett. B 746 (2015) 351 [arXiv: 1501.07413] [INSPIRE].

[94] J. Guo, Z. Kang, P. Ko and Y. Orikasa, Accidental dark matter: Case in the scale invariant local B-L model, Phys. Rev. D 91 (2015) 115017 [arXiv:1502.00508] [INSPIRE].

[95] M.R. Buckley et al., Search for Gamma-ray Emission from Dark Matter Annihilation in the Large Magellanic Cloud with the Fermi Large Area Telescope, Phys. Rev. D 91 (2015) 102001 [arXiv: 1502.01020] [INSPIRE].

[96] K.P. Modak and D. Majumdar, Confronting Galactic and Extragalactic $\gamma$-rays Observed by Fermi-lat With Annihilating Dark Matter in an Inert Higgs Doublet Model, Astrophys. J. Suppl. 219 (2015) 37 [arXiv: 1502.05682] [INSPIRE].

[97] A. Achterberg, S. Amoroso, S. Caron, L. Hendriks, R. Ruiz de Austri and C. Weniger, A description of the Galactic Center excess in the Minimal Supersymmetric Standard Model, JCAP 08 (2015) 006 [arXiv: 1502.05703] [INSPIRE].

[98] T. Gherghetta, B. von Harling, A.D. Medina, M.A. Schmidt and T. Trott, SUSY implications from WIMP annihilation into scalars at the Galactic Center, Phys. Rev. D 91 (2015) 105004 [arXiv: 1502.07173] [INSPIRE].

[99] G. Elor, N.L. Rodd and T.R. Slatyer, Multi-step cascade annihilations of dark matter and the Galactic Center excess, Phys. Rev. D 91 (2015) 103531 [arXiv:1503.01773] [INSPIRE].

[100] J. Kopp, J. Liu and X.-P. Wang, Boosted Dark Matter in IceCube and at the Galactic Center, JHEP 04 (2015) 105 [arXiv: 1503.02669] [INSPIRE].

[101] X.-J. Bi, L. Bian, W. Huang, J. Shu and P.-F. Yin, Interpretation of the Galactic Center excess and electroweak phase transition in the NMSSM, Phys. Rev. D 92 (2015) 023507 [arXiv: 1503.03749] [INSPIRE].

[102] T. Appelquist et al., Stealth Dark Matter: Dark scalar baryons through the Higgs portal, arXiv:1503.04203 [INSPIRE].

[103] A. Rajaraman, J. Smolinsky and P. Tanedo, On-Shell Mediators and Top-Charm Dark Matter Models for the Fermi-LAT Galactic Center Excess, arXiv:1503.05919 [INSPIRE].

[104] J.M. Cline, G. Dupuis, Z. Liu and W. Xue, Multimediator models for the galactic center gamma ray excess, Phys. Rev. D 91 (2015) 115010 [arXiv:1503.08213] [INSPIRE].

[105] K. Ghorbani and H. Ghorbani, Two-portal Dark Matter, Phys. Rev. D 91 (2015) 123541 [arXiv: 1504.03610] [INSPIRE].

[106] P. Ko and Y. Tang, Dark Higgs Channel for FERMI GeV $\gamma$-ray Excess, arXiv: 1504.03908 [INSPIRE].

[107] J. Kim, J.-C. Park and S.C. Park, Galactic center GeV gamma-ray excess from dark matter with gauged lepton numbers, arXiv: 1505.04620 [INSPIRE].

[108] A.D. Banik, D. Majumdar and A. Biswas, Possible Explanation of Indirect Gamma Ray Signatures from Hidden Sector $\mathrm{SU}(2)_{\mathrm{H}}$ Fermionic Dark Matter, arXiv: 1506.05665 [INSPIRE].

[109] L. Calibbi, J.M. Lindert, T. Ota and Y. Takanishi, A lower bound on light neutralino dark matter from LHC data, arXiv: 1405.3884 [INSPIRE].

[110] U. Ellwanger, C. Hugonie and A.M. Teixeira, The Next-to-Minimal Supersymmetric Standard Model, Phys. Rept. 496 (2010) 1 [arXiv:0910.1785] [InSPIRE]. 
[111] J. Cao, C. Han, L. Wu, P. Wu and J.M. Yang, A light SUSY dark matter after CDMS-II, LUX and LHC Higgs data, JHEP 05 (2014) 056 [arXiv: 1311.0678] [INSPIRE].

[112] J. Cao, F. Ding, C. Han, J.M. Yang and J. Zhu, A light Higgs scalar in the NMSSM confronted with the latest LHC Higgs data, JHEP 11 (2013) 018 [arXiv:1309.4939] [INSPIRE].

[113] J.-J. Cao, Z.-X. Heng, J.M. Yang, Y.-M. Zhang and J.-Y. Zhu, A SM-like Higgs near 125 GeV in low energy SUSY: a comparative study for MSSM and NMSSM, JHEP 03 (2012) 086 [arXiv: 1202.5821] [INSPIRE].

[114] K. Griest, M. Kamionkowski and M.S. Turner, Supersymmetric Dark Matter Above the $W$ Mass, Phys. Rev. D 41 (1990) 3565 [inSPIRE].

[115] J.F. Navarro, C.S. Frenk and S.D.M. White, The Structure of cold dark matter halos, Astrophys. J. 462 (1996) 563 [astro-ph/9508025] [INSPIRE].

[116] A. Klypin, H. Zhao and R.S. Somerville, Lambda CDM-based models for the Milky Way and M31 I: Dynamical models, Astrophys. J. 573 (2002) 597 [astro-ph/0110390] [INSPIRE].

[117] G. Bélanger, F. Boudjema and A. Pukhov, MicrOMEGAs: a code for the calculation of Dark Matter properties in generic models of particle interaction, arXiv: 1402.0787 [INSPIRE].

[118] G. Bélanger, F. Boudjema, A. Pukhov and A. Semenov, MicrOMEGAs 3 : A program for calculating dark matter observables, Comput. Phys. Commun. 185 (2014) 960 [arXiv: 1305.0237] [INSPIRE].

[119] G. Bélanger et al., Indirect search for dark matter with MicrOMEGAs2.4, Comput. Phys. Commun. 182 (2011) 842 [arXiv: 1004.1092] [INSPIRE].

[120] G. Bélanger, F. Boudjema, A. Pukhov and A. Semenov, Dark matter direct detection rate in a generic model with MicrOMEGAs 2.2, Comput. Phys. Commun. 180 (2009) 747 [arXiv:0803.2360] [INSPIRE].

[121] G. Bélanger, F. Boudjema, A. Pukhov and A. Semenov, MicrOMEGAs 2.0: A Program to calculate the relic density of dark matter in a generic model, Comput. Phys. Commun. 176 (2007) 367 [hep-ph/0607059] [INSPIRE].

[122] T. Sjöstrand et al., An Introduction to PYTHIA 8.2, Comput. Phys. Commun. 191 (2015) 159 [arXiv: 1410.3012] [INSPIRE].

[123] U. Ellwanger, J.F. Gunion and C. Hugonie, NMHDECAY: A Fortran code for the Higgs masses, couplings and decay widths in the NMSSM, JHEP 02 (2005) 066 [hep-ph/0406215] [INSPIRE].

[124] U. Ellwanger and C. Hugonie, NMHDECAY 2.0: An Updated program for sparticle masses, Higgs masses, couplings and decay widths in the NMSSM, Comput. Phys. Commun. 175 (2006) 290 [hep-ph/0508022] [INSPIRE].

[125] G. Degrassi, S. Heinemeyer, W. Hollik, P. Slavich and G. Weiglein, Towards high precision predictions for the MSSM Higgs sector, Eur. Phys. J. C 28 (2003) 133 [hep-ph/0212020] [INSPIRE].

[126] J. Cao and J.M. Yang, Anomaly of $Z b_{\bar{b}}$ coupling revisited in MSSM and NMSSM, JHEP 12 (2008) 006 [arXiv:0810.0751] [INSPIRE].

[127] P. Bechtle, O. Brein, S. Heinemeyer, G. Weiglein and K.E. Williams, HiggsBounds: Confronting Arbitrary Higgs Sectors with Exclusion Bounds from LEP and the Tevatron, Comput. Phys. Commun. 181 (2010) 138 [arXiv:0811.4169] [InSPIRE]. 
[128] P. Bechtle, O. Brein, S. Heinemeyer, G. Weiglein and K.E. Williams, HiggsBounds 2.0.0: Confronting Neutral and Charged Higgs Sector Predictions with Exclusion Bounds from LEP and the Tevatron, Comput. Phys. Commun. 182 (2011) 2605 [arXiv:1102.1898] [INSPIRE].

[129] T. Stefaniak, Recent Developments in HiggsBounds and a Preview of HiggsSignals, PoS (CHARGED 2012) 024.

[130] P. Bechtle et al., HiggsBounds - 4: Improved Tests of Extended Higgs Sectors against Exclusion Bounds from LEP, the Tevatron and the LHC, Eur. Phys. J. C 74 (2014) 2693 [arXiv: 1311.0055] [INSPIRE].

[131] P. Bechtle, S. Heinemeyer, O. Stål, T. Stefaniak and G. Weiglein, HiggsSignals: Confronting arbitrary Higgs sectors with measurements at the Tevatron and the LHC, Eur. Phys. J. C 74 (2014) 2711 [arXiv:1305.1933] [INSPIRE].

[132] K. Griest and D. Seckel, Three exceptions in the calculation of relic abundances, Phys. Rev. D 43 (1991) 3191 [INSPIRE].

[133] C. Han, Probing light bino and higgsinos at the LHC, arXiv:1409.7000 [INSPIRE].

[134] Fermi-LAT collaboration, M. Ackermann et al., Search for gamma-ray spectral lines with the Fermi large area telescope and dark matter implications, Phys. Rev. D 88 (2013) 082002 [arXiv: 1305.5597] [INSPIRE].

[135] ATLAS collaboration, Observation of a new particle in the search for the Standard Model Higgs boson with the ATLAS detector at the LHC, Phys. Lett. B 716 (2012) 1 [arXiv: 1207.7214] [INSPIRE].

[136] CMS collaboration, Observation of a new boson at a mass of $125 \mathrm{GeV}$ with the CMS experiment at the LHC, Phys. Lett. B 716 (2012) 30 [arXiv:1207.7235] [INSPIRE].

[137] J.F. Gunion, Y. Jiang and S. Kraml, Could two NMSSM Higgs bosons be present near 125 GeV?, Phys. Rev. D 86 (2012) 071702 [arXiv:1207.1545] [INSPIRE].

[138] J.F. Gunion, Y. Jiang and S. Kraml, Diagnosing Degenerate Higgs Bosons at $125 \mathrm{GeV}$, Phys. Rev. Lett. 110 (2013) 051801 [arXiv:1208.1817] [INSPIRE].

[139] http://dmtools.brown.edu/.

[140] G. Jungman, M. Kamionkowski and K. Griest, Supersymmetric dark matter, Phys. Rept. 267 (1996) 195 [hep-ph/9506380] [INSPIRE].

[141] J. Cao, D. Li, L. Shang, P. Wu and Y. Zhang, Exploring the Higgs Sector of a Most Natural NMSSM and its Prediction on Higgs Pair Production at the LHC, JHEP 12 (2014) 026 [arXiv: 1409.8431] [INSPIRE].

[142] J. Kozaczuk and T.A.W. Martin, Extending LHC Coverage to Light Pseudoscalar Mediators and Coy Dark Sectors, JHEP 04 (2015) 046 [arXiv: 1501.07275] [INSPIRE]. 